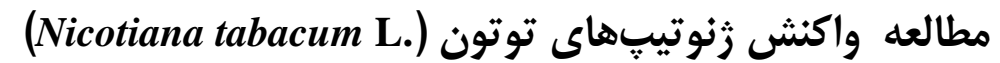

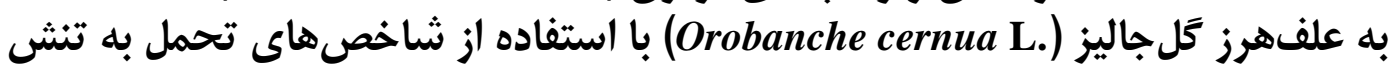

\author{
رضا درويشزاده' و ايرج برنوسى \\ ا- استاد كروه اصلاح و بيوتكنولوزى كياهى دانشكده كشاورزى دانشخاه اروميه و استاد يُوهشكده زيست فناورى دانشكاه اروميه،

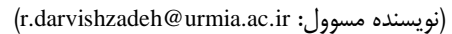

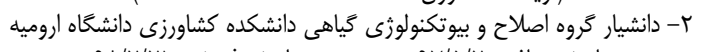 \\ تاريخ دريافت: \\ صفحه: بوا تا IVT
}

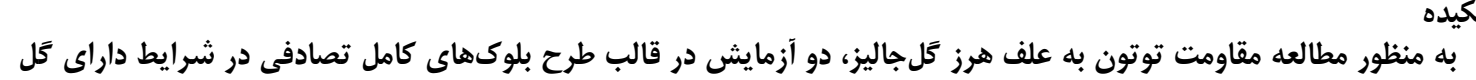

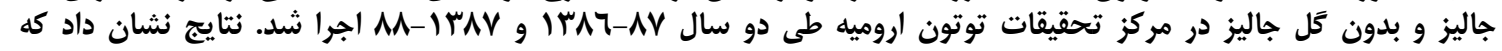

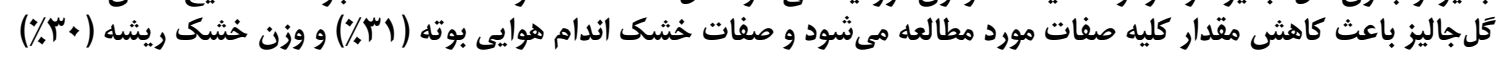

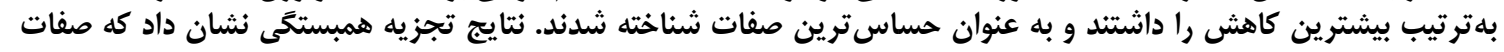

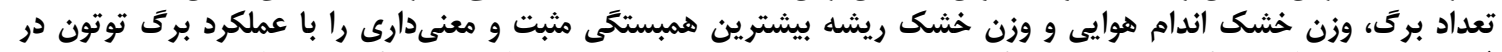

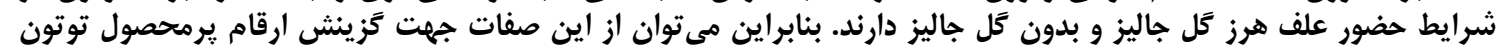

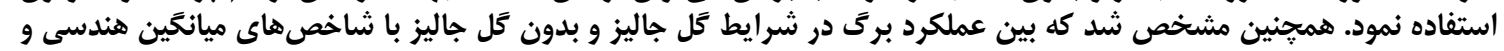

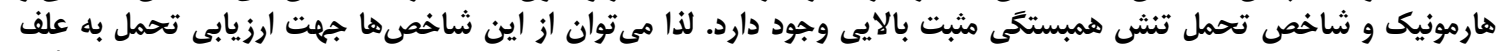

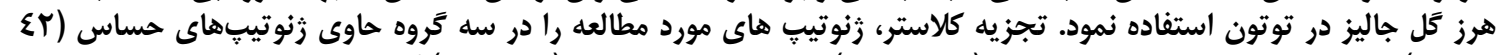

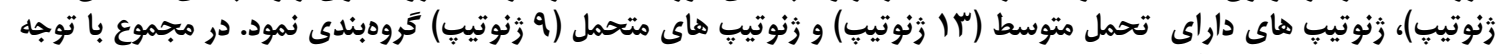

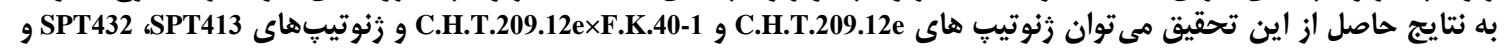

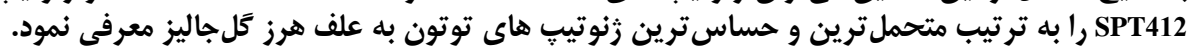

$$
\text { وازههاى كليدى: توتون شرقى، تجزيه كلاستر، علف هرز يارازيت، شاخص تحمل، عملكرد برتى }
$$

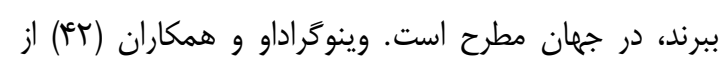

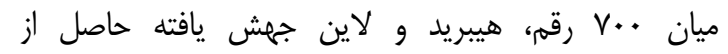

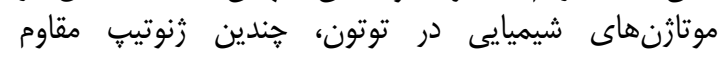
،Trabzon-258 CCorojo ،Ns-2512،Hiks-Resistant) (T×R Ghatana ،Gamaica-K-4442 Oramosa Orobanche aegyptiaca

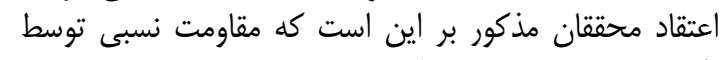

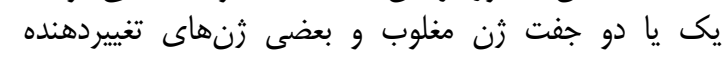

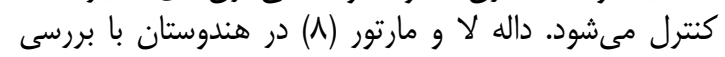

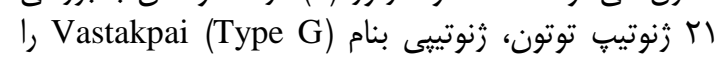

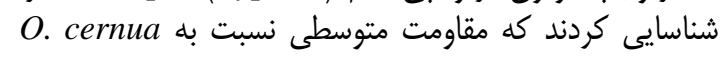

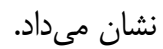

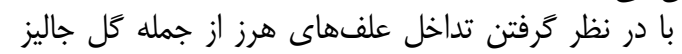

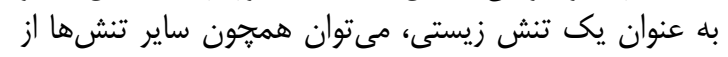

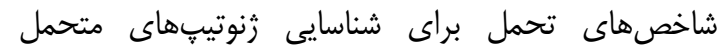

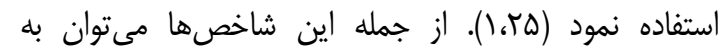

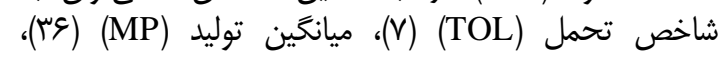

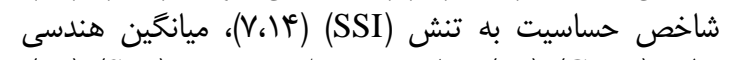

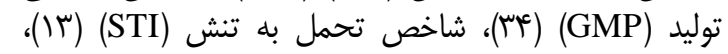

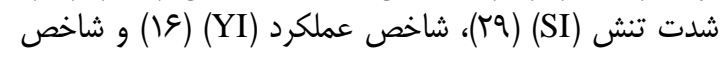

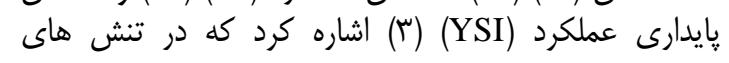

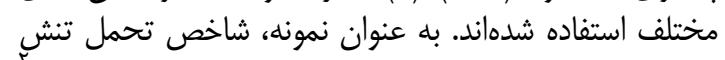

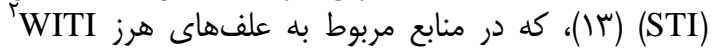

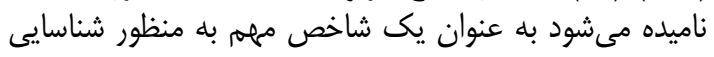

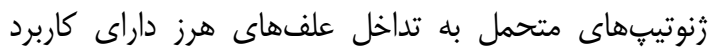

مقدمه

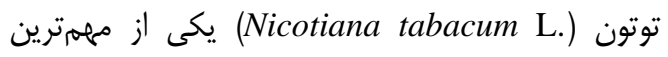

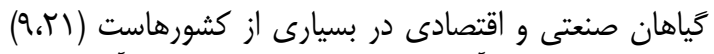

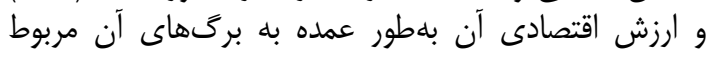

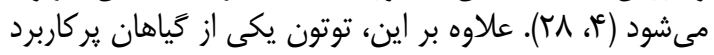

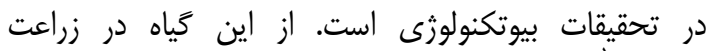

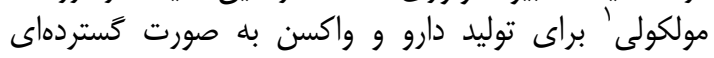

استفاده مى شود (ه). (ه).

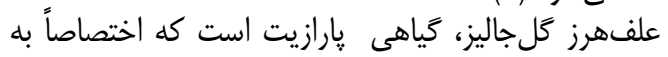

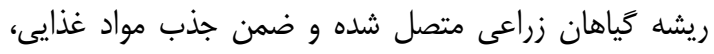

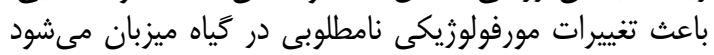

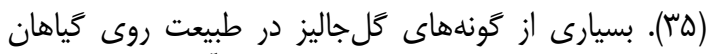

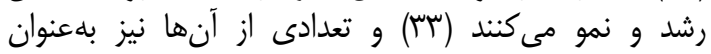

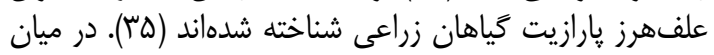

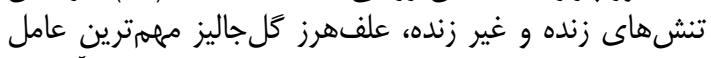

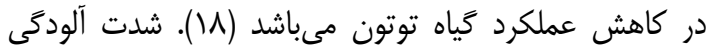

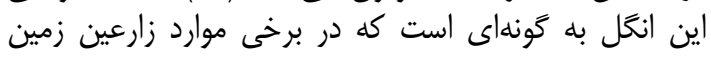

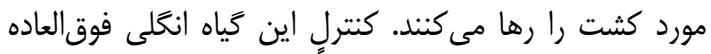

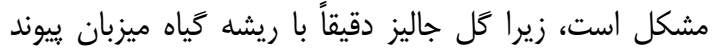

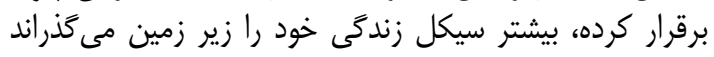

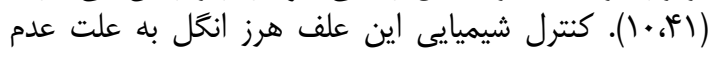

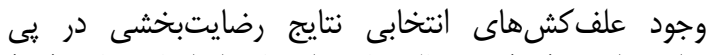

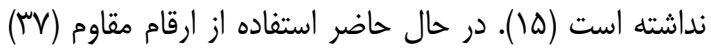

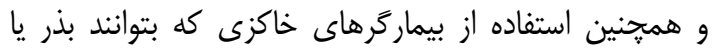

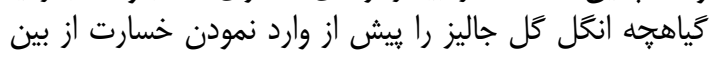


براى انجام آزمايش در هر دو شرايط از كَلدانهاى سفالى

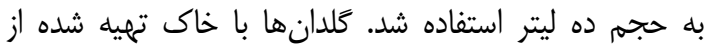

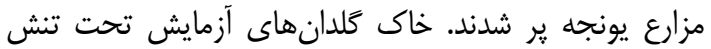

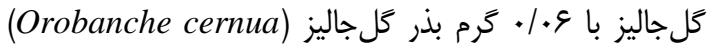

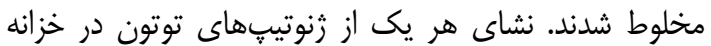

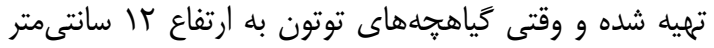

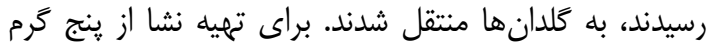

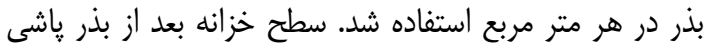

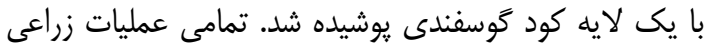

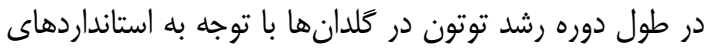

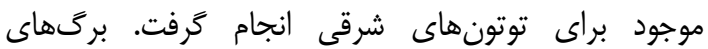

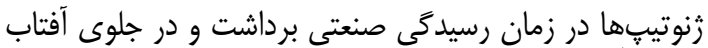

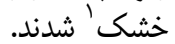

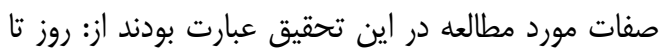

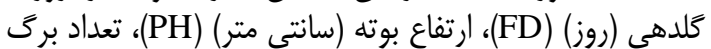

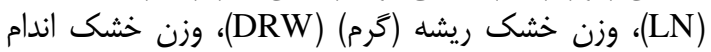

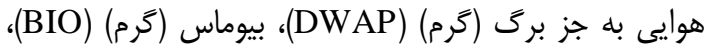

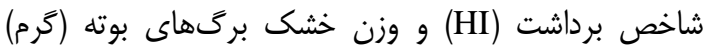

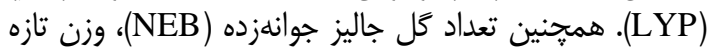

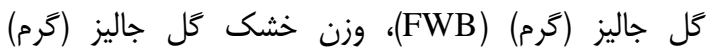
(DWB)

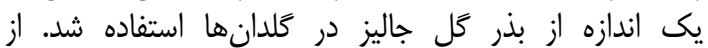

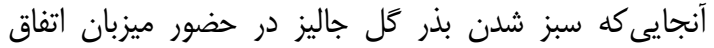

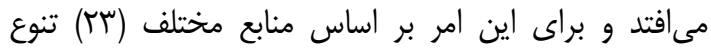

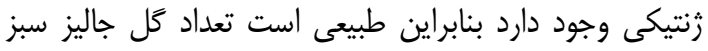

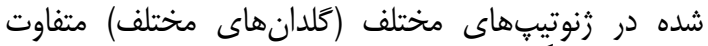

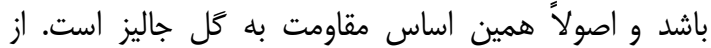

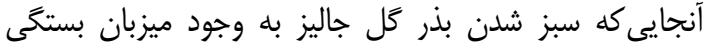

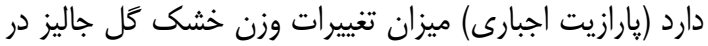

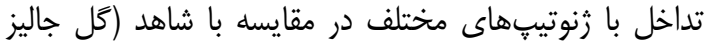
بدون توتون) قابل محاسبه نبود.

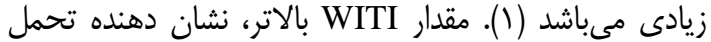

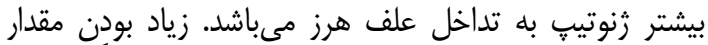

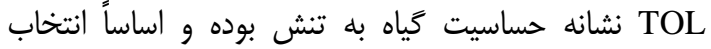

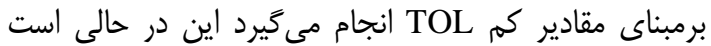

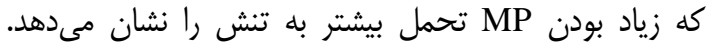

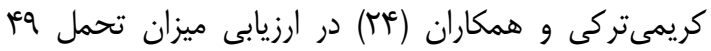

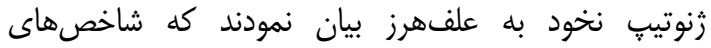

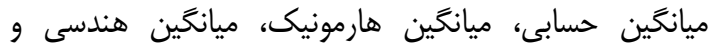

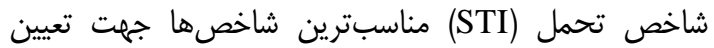

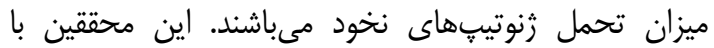

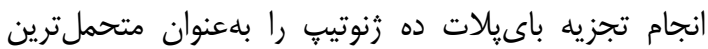

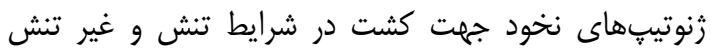
معرفى نمودند. اين تحقيق به منظور تعيين معيارى مناسب جهت كزئ زينش

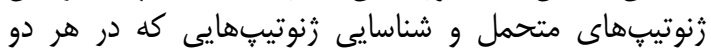

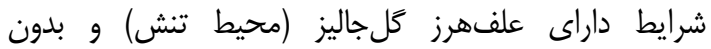

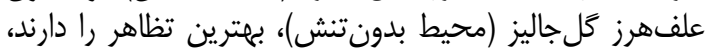
صورت كرفته است.

\section{مواد و روشها}

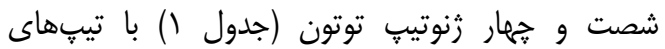

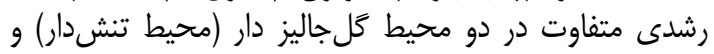

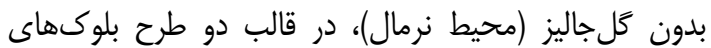

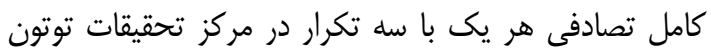

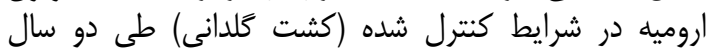

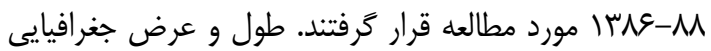

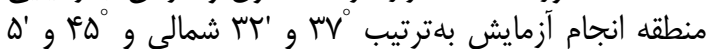

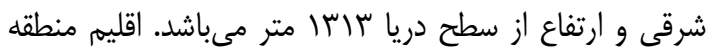

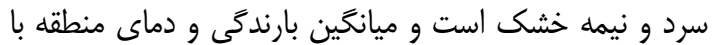

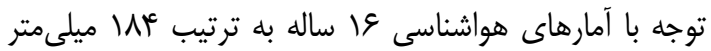

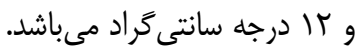

جدول (- اسامى زنوتيبهاى توتون مورد مطالعه

Table 1. Name of studied tobacco genotypes

\begin{tabular}{|c|c|c|c|c|c|c|c|c|c|c|}
\hline 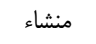 & رنوتيب & منشاء كد & رنوتي & 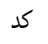 & 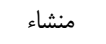 & رنوتيب & 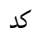 & 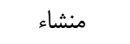 & ز & كد \\
\hline Iran & SPT 403 & 49 Iran & C.H.T.209.12exF.K.40-1 & 33 & - & K. B & 17 & Turkey & Kharmanli163 & 1 \\
\hline Iran & SPT 408 & 50 Iran & С.Н.T.266-6 & 34 & Bulgaria & G.D. 165 & 18 & Bulgaria & Nevrokop & 2 \\
\hline Iran & SPT 412 & 51 Iran & С.Н.T.283-8 & 35 & - & H.T. I & 19 & Turkey & Trabozan & 3 \\
\hline Iran & Isfehan5 & 52 Iran & C.H.T.273-38 & 36 & Bulgaria & $\underset{659}{\text { Kramograd N.H.H. }}$ & 20 & Bulgaria & Krumovgraid & 4 \\
\hline Iran & SPT 432 & 53 Iran & Basma12-2 & 37 & - & T.K.23 & 21 & Greece & Basma S. 31 & 5 \\
\hline Iran & SPT 433 & 54 Iran & Basma16-10 & 38 & Iran & L16 & 22 & Yugoslavia & Triumph & 6 \\
\hline Iran & SPT 434 & 55 Iran & Basma104-1 & 39 & Turkey & Izmir 7 & 23 & Iran & Matianus & 7 \\
\hline Iran & SPT 439 & 56 Iran & Basma181-8 & 40 & Russian & Pobeda 1 & 24 & - & Immni3000 & 8 \\
\hline Iran & SPT 441 & 57 Iran & P.D. 325 & 41 & - & Rustica & 25 & - & Tyk-Kula & 9 \\
\hline Iran & SPT 413 & 58 Iran & P.D.406 & 42 & Turkey & Samsun 1 & 26 & - & Ss-289-2 & 10 \\
\hline Bulgaria & L17 & 59 Iran & P.D. 328 & 43 & Turkey & Samsun 959 & 27 & Yugoslavia & Ohdaruma & 11 \\
\hline Iran & Balouch & 60 Iran & P.D.329 & 44 & Turkey & Samsun Katerini & 28 & - & T.B.22 & 12 \\
\hline Iran & Saderati & 61 Iran & P.D.336 & 45 & Iran & OR-205 & 29 & Iran & Alborz23 & 13 \\
\hline Iran & Shahroudi & 62 Iran & P.D. 345 & 46 & Iran & OR-345 & 30 & Iran & F.K. $40-1$ & 14 \\
\hline- & T.K. L & 63 Iran & P.D. 371 & 47 & Iran & OR-379 & 31 & Bulgaria & PI7 & 15 \\
\hline Iran & C.Н.T.269-12e & 64 Iran & P.D.381 & 48 & Iran & С.Н.Т.209.12e & 32 & - & K.P.На & 16 \\
\hline
\end{tabular}

1- Sun-cured 

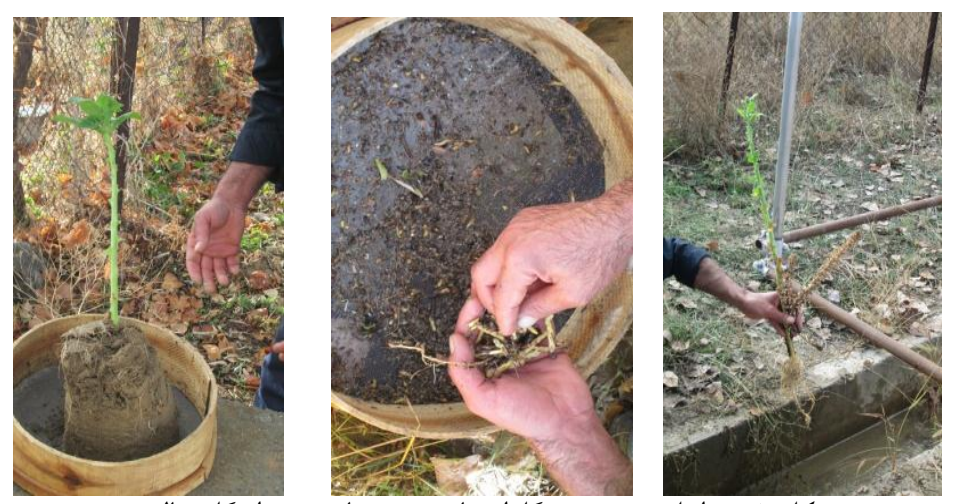

شكل ا- مراحل شستشوى كَلدانها جهت شمارش تعداد كل جاليز

Figure 1. Pots washing steps in order to counting the number of Orobanche

بين شاخصهاى تحمل و عملكرد برك توتون در شرايط

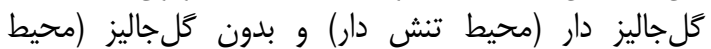

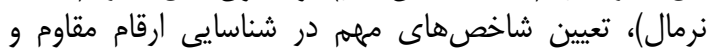

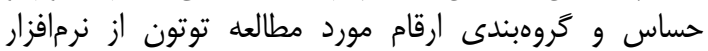
نسخه آ (••) استفاده كَرديد. SPSS
در ادامه بلهنظور تعيين ارقام متحمل و حساس بله

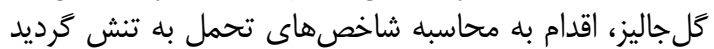

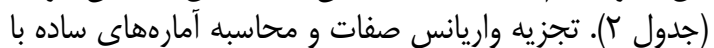
استفاده از نرمافزار SAS نسخه

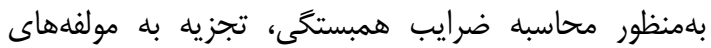

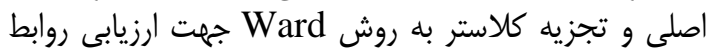

جدول r- شاخصهاى متحمل به تنش استفاده شده براى ارزيابى واكنش زنوتيڤهاى توتون به علفهرز كَ جاليز Table 2. Stress tolerance indices used for evaluating the reaction of tobacco genotypes to Orobanche

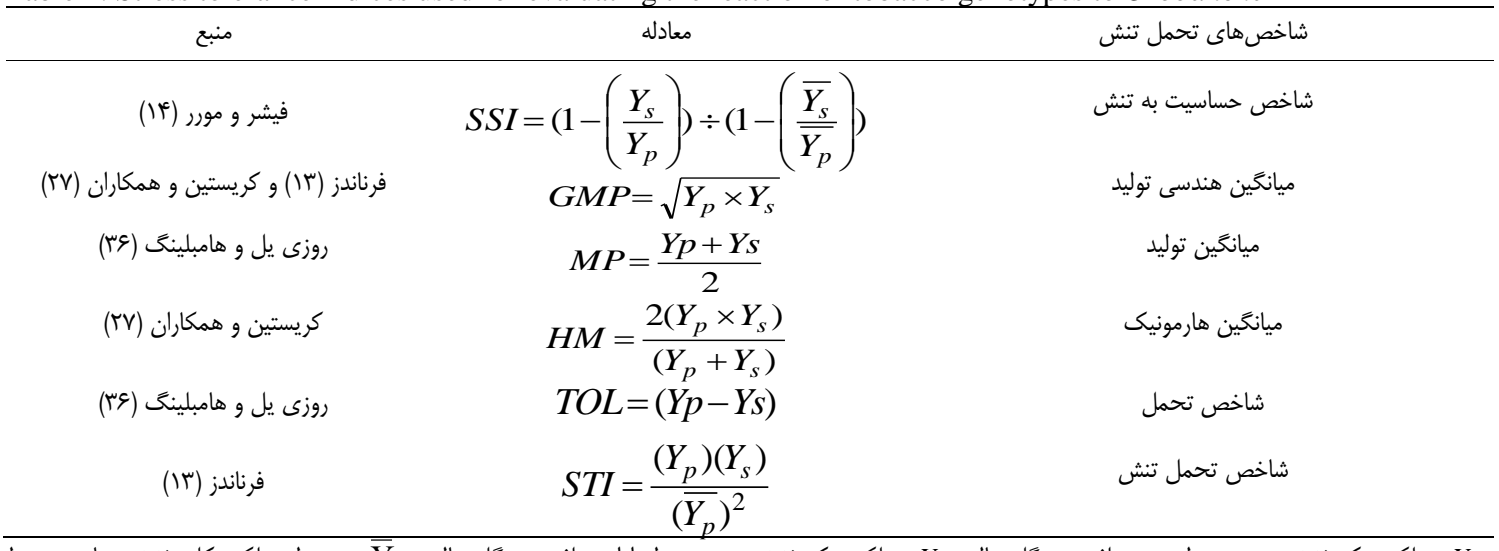

بp بدون علف هرز كل جاليز.

ارقام باشد كه اين امر زمينه را براى انتخاب متحملترين ارقام

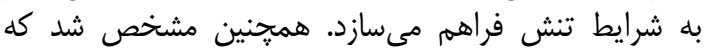

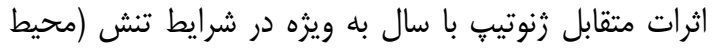

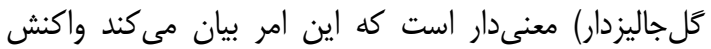

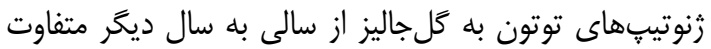

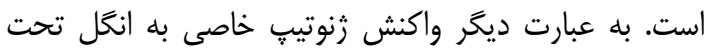

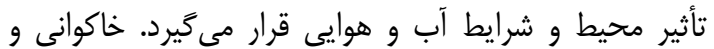

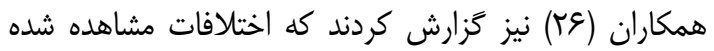

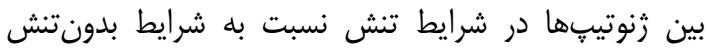

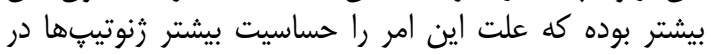

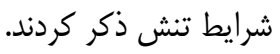

تجزيه واريانس صفات در شرايط بدون

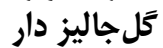

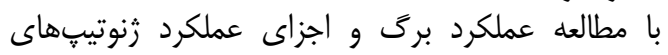

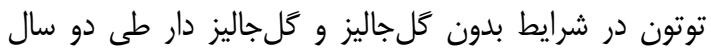
زراعى مشخص شد كه بين ثرنوتيبها

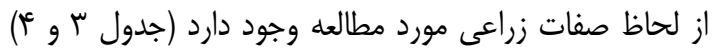

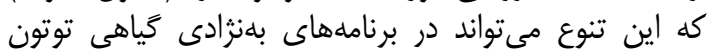

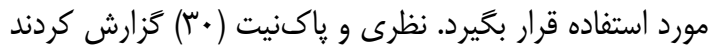

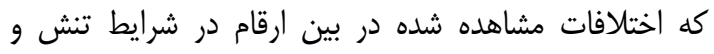
بدون تنش مىتواند ناشى از وجود تنوع زُنتيكى بالا در بين آنش 
جدول س- تجزيه واريانس صفات مطالعه شده در توتون در شرايط نرمال (بدون علفهرز گَل جاليز) Table 3. Variance analysis of studied traits in tobacco under normal conditions

\begin{tabular}{|c|c|c|c|c|c|c|c|c|c|}
\hline \multicolumn{8}{|c|}{ ميانخين مربعات } & \multirow{2}{*}{ آل آرجى } & \multirow[b]{2}{*}{ منابع تغييرات } \\
\hline بركَهاي بوته (كرم) & شراشت & بيوماس (كرم) & وزنه خز برى اندام هوايى & ريشه (كرم) خشكى & تعداد برى & ارتفاع بوته & $\begin{array}{c}\text { روز تا كلدهى } \\
\text { (روز) }\end{array}$ & & \\
\hline$\Delta T \Delta / \Gamma^{*}$ & $4 \Lambda^{4} / 9^{*}$ & $1.94 / 1^{\mathrm{ns}}$ & $\mathrm{N} / \mathrm{r}^{\mathrm{nS}}$ & $r q / r^{n s}$ & $9 / 4^{\mathrm{ns}}$ & $1 \cdot \Delta / /^{\prime \prime}$ & $r / a^{n s}$ & 1 & سال \\
\hline$r \varepsilon / \Delta$ & $r r / q$ & $r \mid F / T$ & $\Delta \Gamma / 1$ & $I V / F$ & $1 /$. & $r / 9$ & $1 / 1$ & r & اشتباه 1 \\
\hline 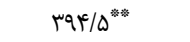 & 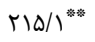 & $19 \mu \cdot 10^{* * *}$ & $r q \cdot 1 \Lambda^{* * *}$ & 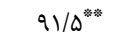 & $|9 N| \cdot{ }^{* * *}$ & 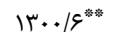 & $r \Delta{ }^{\prime} /^{* *: *}$ & س & رنوتي؟ \\
\hline$\Lambda \in / 1^{* * *}$ & $q \vee / \mathrm{V}^{* * *}$ & $T r N / D^{n s}$ & $s \mu / r^{\mathrm{ns}}$ & $r M / r^{\mathrm{ns}}$ & $11 / \wedge^{\mathrm{ns}}$ & $\Lambda V / \Lambda^{\mathrm{ns}}$ & $\mid \varepsilon / 8^{* * *}$ & r & رنوتي״ × سال \\
\hline$\Delta T / 1$ & $\Delta T / \Lambda$ & $T I T / \Delta$ & D) $/ \mathrm{C}^{\mathrm{C}}$ & $I V / T$ & $r \omega / \Lambda$ & $1 . r / \pi$ & $r / s$ & TFD & اشتباه r | ك \\
\hline i $1 / \Lambda$ & $r \cdot / 9$ & r/r & ז & זN/V & $r / \mu$ & $1 \omega / V$ & $r / \Delta$ & --- & درصد ضريب تغييرات \\
\hline
\end{tabular}

جدول ع- تجزيه واريانس صفات مطالعه شده در توتون در شرايط تنش (محيط كل جاليز دار) Table 4. Variance analysis of studied traits in tobacco under Orobanche stress conditions

\begin{tabular}{|c|c|c|c|c|c|c|c|c|c|}
\hline \multicolumn{8}{|c|}{ ميانگين مربعات } & \multirow{3}{*}{ آزادى } & \multirow[t]{3}{*}{ منابع تغييرات } \\
\hline وزن خشك & شاخص & بيوماس & وزن خشك اندام هوايى & وزن خشك & تعداد & ارتفاع بوته & روز تا كلدهى & & \\
\hline بركَهاى بوته (كرم) & برداشت & $(5)$ & به جز برى (كرم) & ريشه (كرم) & برى & (سانتى متر) & $(j g)$ & & \\
\hline$M I T N / q^{* *}$ & $1 \cdot \Delta T \backslash / \Lambda^{* * *}$ & $\| f^{\prime} \Delta / /^{* * *}$ & $11 \cdot Y g / V^{*}$ & $g^{\mathrm{r}} \Delta / \varphi^{*}$ & $T r \Delta / q^{*}$ & TYYT/ & $11 \% / 9^{\mathrm{ns}}$ & 1 & سال \\
\hline N/ & $I V / T$ & $r M / r$ & $\Delta \& M / 1$ & $9 \cdot / V$ & TN/T & ए११/। & $19 / 8$ & r & | اشتباه 1 \\
\hline ५৭৭// & 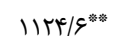 & $r \cdot 9 / q^{* * * *}$ & $r \Delta \cdot N / r^{*: *}$ & YAT/V $/ V^{\text {*ै: }}$ & 满 & 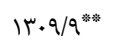 & $r \Delta Q / *^{* * * *}$ & G & كتيّ \\
\hline $1 \wedge \mathrm{V} / \mathrm{A}^{* * * *}$ & $\uparrow \wedge \Delta / \Lambda^{* * * *}$ & $\Lambda \& / \Lambda^{* * *}$ & $911 / \Delta^{* * *}$ & $\mathrm{VN} / \cdot^{\mathrm{ns}}$ & $r \mid / s^{\mathrm{ns}}$ & $r \mu r / V^{\text {wis }}$ & $\mathrm{mF} / \mathrm{q}^{* \mathrm{w}^{*}}$ & gr & رثنوتي × سال \\
\hline$M N$ & $1 . \Delta / f$ & TN/q & $r \Delta q / V$ & $9 D / V$ & $19 / \Delta$ & $\pi \cdot / g$ & $1 \% / 9$ & TED & اشتباه r \\
\hline$r T / r$ & $m /{ }^{e}$ & $\mathrm{~F} / \mathrm{A}$ & ( & $r / \Delta$ & $r \cdot / V$ & $19 / 8$ & s/T & --- & درصد ضريب تغييرات \\
\hline
\end{tabular}

مى يابند. بررسى ضريب تغييرات فنوتييى (جدول ه) نيز نشان

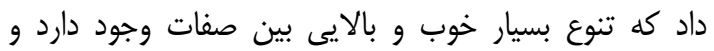

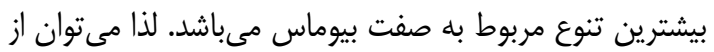

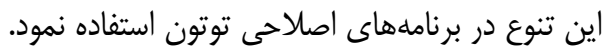

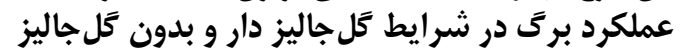

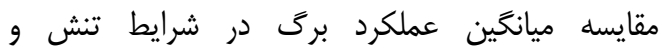

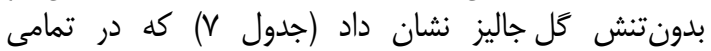
زنوتيبها عملكرد در شرايط تنش در مقايسه با شرايط بدونتش مائش

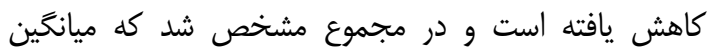

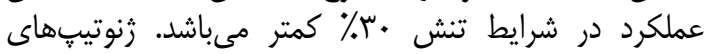
و C.H.T.209.12exF.K.40-1 ب.H.T.I C.H.T.209.12e

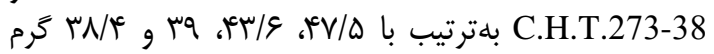

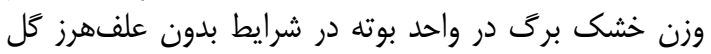

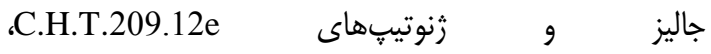

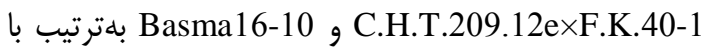

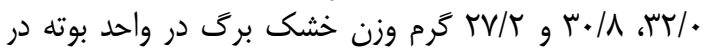

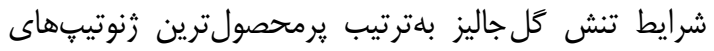

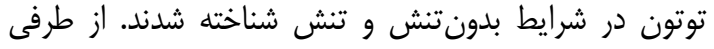

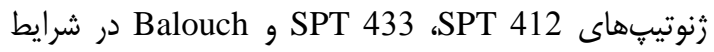

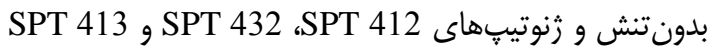

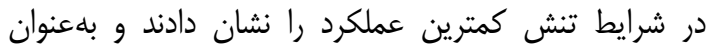

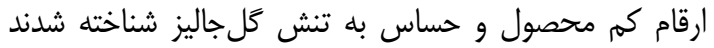

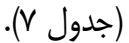

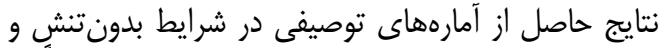

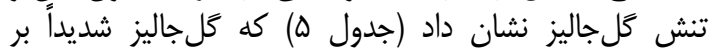

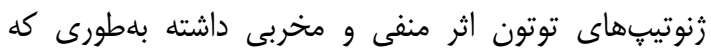

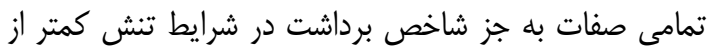

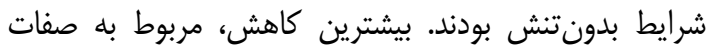

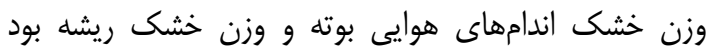

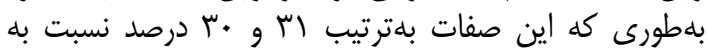

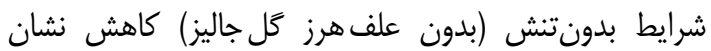

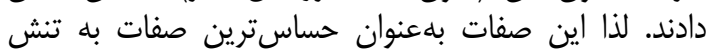

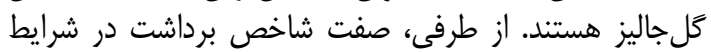

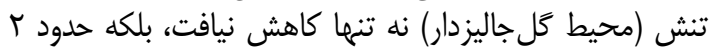

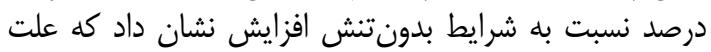

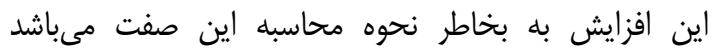

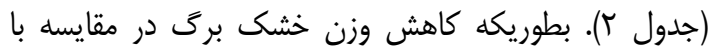

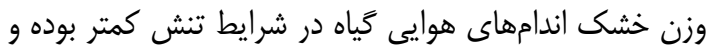

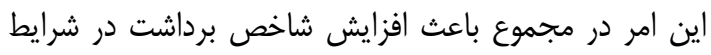

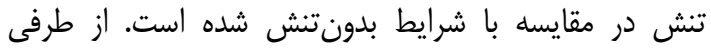

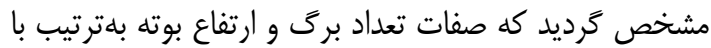

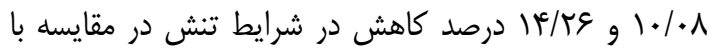

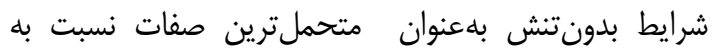

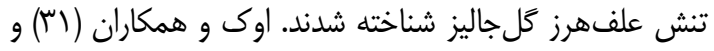

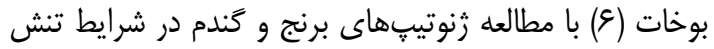

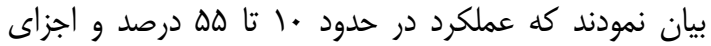

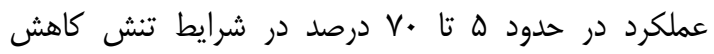


جدول ه- آمارههاى ساده صفات زراعى زنوتييهاى توتون در شرايط بدونتش و تنش كل جاليز Table 5. Simple statistic parameters of agronomical traits of tobacco genotypes under normal and orobanche stress

\begin{tabular}{|c|c|c|c|c|c|c|c|c|c|c|c|}
\hline \multicolumn{2}{|c|}{ ضريب تغييرات } & \multirow[t]{2}{*}{ ضرب كاهش } & \multicolumn{2}{|c|}{ انحراف معيار 土 ميانخين } & \multicolumn{2}{|c|}{ دامنه } & \multicolumn{2}{|c|}{ 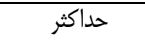 } & \multicolumn{2}{|c|}{ 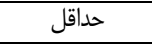 } & \multirow[t]{2}{*}{ صفات } \\
\hline تنش & نرمال & & 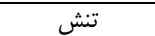 & نرمال & تنش & نرمال & تنش & نرمال & تنش & نرمال & \\
\hline $9 / T$ & $r / \Delta$ & $\cdot / \mathrm{N}^{c}$ & $q \cdot / f^{*} \Delta \pm q / \Delta f^{f}$ & $9 \cdot / 9 \cdot \pm V / 9 \mathrm{~V}$ & $\mu \nu / \cdot$ & $4 \cdot / \cdot$. & $V^{\mu / \mu} / \mu$ & $\Lambda \cdot / \Lambda r^{c}$ & IT/N & $\varphi \cdot / \Lambda \mu$ & إهى (روز) \\
\hline $19 / 9$ & $10 / V$ & $1 F / r 9$ & $\Delta \Delta / \wedge \Delta \pm 1 \Delta / 1$. & $\varepsilon D / / F \pm \mid N / \cdot \Delta$ & $\Delta V / \Gamma$ & $q . / 1 \%$ & $\Lambda F / \mu$ & $11 \pi / r$. & $r V / .$. & $r \mu . \varphi$ & ارتفاع بوته (سانتى متر) \\
\hline$r \cdot / r$ & $r M / r$ & $1.1 \cdot 1$ & $M / /{ }^{*} \Delta \pm \varepsilon / M^{C}$ & 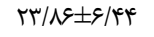 & $\mu \% /$. & $r q / v \Delta$ & $\mu$ & $f \cdot / V$ & $9 / .$. & $11 / . r$ & تعداد برى \\
\hline$r r / \Delta$ & $r Y / q$ & $r \cdot / 19$ & $V / \Delta \cdot \pm r / 99$ & $1 \cdot / V F \pm r / V \Delta$ & $I T / N$ & $19 / 19$ & $\mid Q / \cdot r$ & $T T / / Q$ & $r / 9$. & $r / r \varepsilon$ & وزن خشك ريشه (كرم) \\
\hline r & $M T / r$ & $r / / r$ & $\mid r / Q \varepsilon \pm g / K \digamma$ & $|N / \Lambda| \pm N / \cdot 9$ & $r N / \cdot T$ & 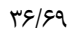 & $M T / \Delta F$ & $4 \pi / v q$ & $r / \Delta T$ & $\mathrm{~V} / \mathrm{l}$ & وزن خشى اندام هوايى به جزء برى (كرم) \\
\hline$<1 / \wedge$ & $r \cdot / q$ & $r q / / 4$ & $r r / \Lambda q \pm I F / \Delta F$ & $\varphi \varepsilon|\Lambda| \pm r \cdot 1.9$ & $81 / \mu$. & $\Lambda \mu / \& \Lambda$ & $V T / / Q$ & $1 \cdots / 1 \mid$ & $1 \cdot / \Lambda \Delta$ & $\mid V / I T$ & بيوماس (كرم) \\
\hline$r \mid / \mu^{c}$ & $+1 / \Lambda$ & $-T / T)$ & $r \omega / g r \pm V / f \mathscr{V} V$ & $\mu F / \wedge \Delta \pm V / r q$ & 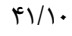 & ro/s & $\Delta N / A T^{F}$ & $\Delta S / T V$ & $I V / V T^{c}$ & $r \cdot / 9$. & شاخص برداشت \\
\hline$r T / r$ & $\lceil N / 1$ & $r q / \mu$ & $|r / r \cdot \pm V / \mu|$ & $\mid V / r g \pm q / \wedge q$ & 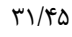 & ו ו & $\mu F / M$ & $\mid \mathrm{F} / \mathrm{qq}$ & 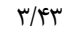 & $r / r q$ & وزن خشك برَّهاى بوته (كرم) \\
\hline
\end{tabular}

${ }^{\mathrm{a}} \mathrm{D}=\frac{\overline{\mathrm{X}}_{\text {Normal }}-\overline{\mathrm{X}}_{\text {Biotic }}}{\overline{\mathrm{X}}_{\text {Normal }}} \times 100$

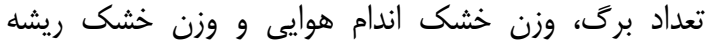

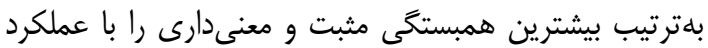

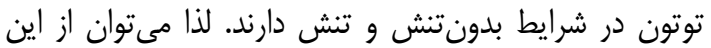

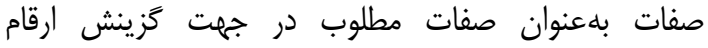
يرمحصول توتون استفاده نمود.

\section{تجزيه همبستكى صفات زراعى}

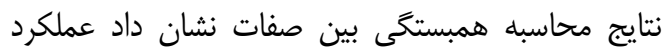

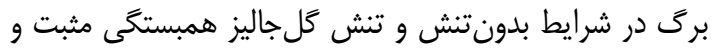

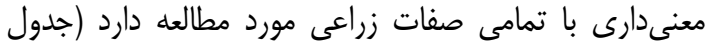

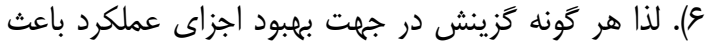

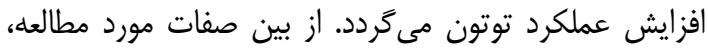

جدول צ- ضرايب همبستخى بين صفات زراعى در زنوتيِهاى توتون در شرايط بدونتنش (زير قطر) و شرايط تنش كلجاليز (بالاى قطر) Table 6. Correlation coefficients between agronomic traits of tobacco genotypes under normal (below diagonal) and

\begin{tabular}{|c|c|c|c|c|c|c|c|c|}
\hline وزن خشك بركهاى & برداشت & 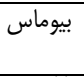 & وزن خشك اندام هوايى به & وزن خشك & تعداد برى & ارتفاع بوته & 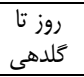 & صفات \\
\hline$\cdot / \Lambda^{* * * * *}$ & . &.$/ \% q^{* 3 *}$ & $\cdot / \Gamma \Lambda^{*}$ &.$/ q^{* * *}$ & $\cdot|\Delta|^{*}$ &.$/\left.9\right|^{* * * i n}$ & $1 / \cdot \cdot$ & روز تا كلدهى (روز) \\
\hline • & -.1 .0 & $\cdot / \wedge^{4} \Lambda^{* * *}$ & $\cdot / 0 \cdot{ }^{* * *}$ & $\cdot / \Delta \varphi^{* * *}$ & $\cdot / 9 \Lambda^{* * * 4}$ & $1 / \cdot$ & $\cdot / \Delta Q^{* * *}$ & ارتفاع بوته (سانتى متر) \\
\hline$\cdot / \Delta \mathrm{V}^{* *:}$ &.$/ \mathrm{V}$ &.$/ 9 \Lambda^{* * a}$ & 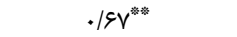 & $\cdot \mid \Delta \gamma^{* * *}$ & $1 / \cdot$. & $\cdot / V Y^{* * a k}$ & $\cdot / \Delta \Lambda^{* * *}$ & تعداد برى \\
\hline$\cdot \mid q^{4}$ &.$/ 1 r$ &.$/ V^{* * m}$ & $\cdot \mid \Delta \xi^{* * * *}$ & $1 / \cdot \cdot$ & 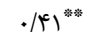 &.$/ 4 q^{* * *: *}$ & $\cdot / r F$ & وزن خشك ريشه (كرم) \\
\hline$\cdot / r^{w * * *}$ &.$/ M$ & $\cdot / 19^{* * *}$ & $1 / \cdot$ & $\cdot \mid \Delta \delta^{* * *}$ & $.19 \Lambda^{* *: *}$ & 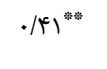 & $\cdot / T v^{*}$ & وزن خشك اندام هوايى به جزء \\
\hline 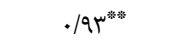 &.$/ 40^{* *}$ & $1 /$. & $\cdot / M^{* * * *}$ & $\cdot / N{ }^{*}$ & • & 年 &.$/ \Gamma \phi^{* *: *}$ & بيوماس (كرم) \\
\hline$\cdot / M^{* * *}$ & $1 / \cdot \cdot$ &.$/ \mu^{*}$ & $\cdot / \cdot v$ & $-. \cdot / \cdot v$ & $\cdot / \cdot 1$ &.$- / 1 f$ &.$/ 10$ & شاخص برداشت \\
\hline $1 / \cdot$ & $.190^{* * *}$ & $. / 9)^{* *: 4}$ & $\cdot / 9 \mathrm{~V}^{* * *}$ & $\cdot \mid \Delta \mu^{* * * *}$ & $\cdot / 0 \cdot *$ & $. / 4)$ & 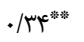 & وزن خشى برگهاى بوته (كرم) \\
\hline
\end{tabular}

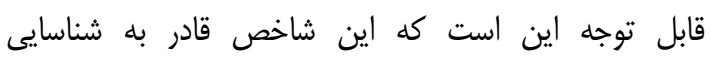

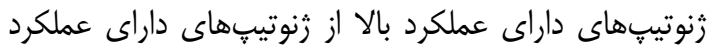

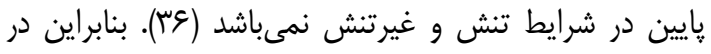

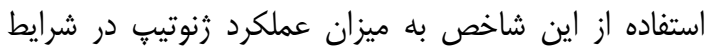

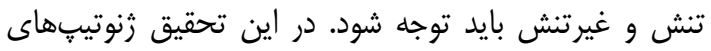

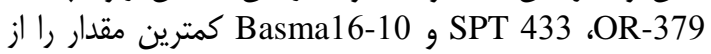

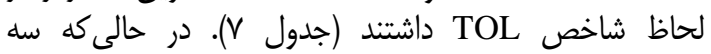
خنوتي عملكرد بالايى در شرايط بدون إنتش نشان دادند ولى به دليل

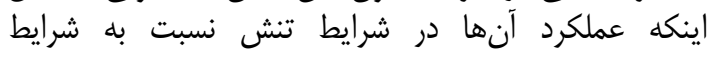

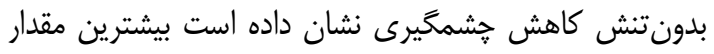

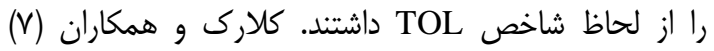

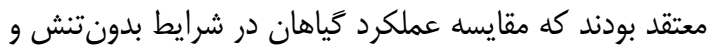

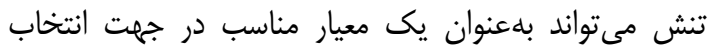

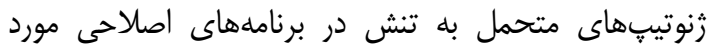
استفاده قرار بخيرد.
تنوع فتوتيبى كَل جاليز

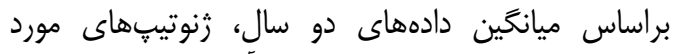

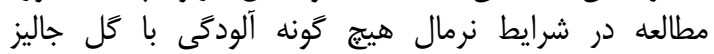

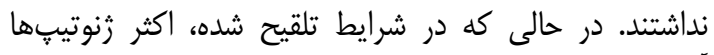

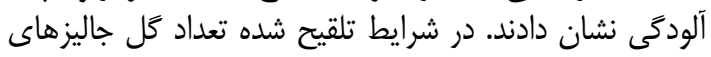

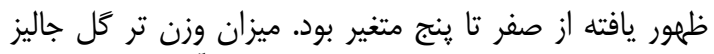

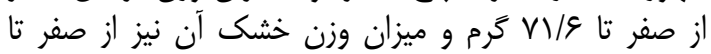

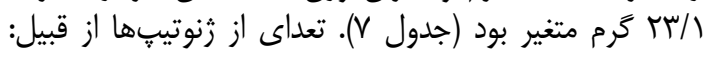
C.H.T.266-6 , C.H.T.209.12e ،G.D.165 ،H.T.I كمترين تعداد كَل جاليز ظهور يافته را به خود اختصاص دادند.

شاخص TOL شاخص TOL از اختلاف عملكرد زنوتيب در شرايط

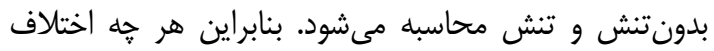

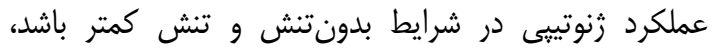

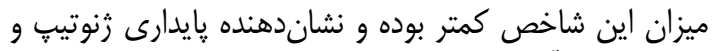

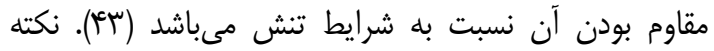




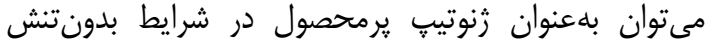

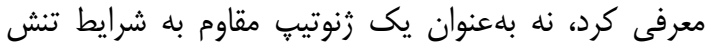

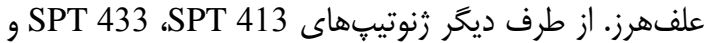

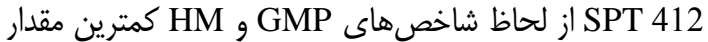

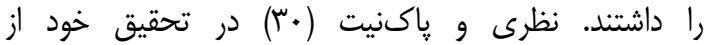

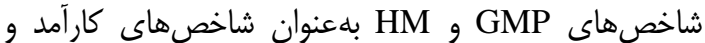
قوى در شناسايى زنوتيبهاى مطلوب و نامطلوب در شرايط

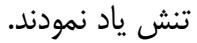

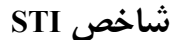

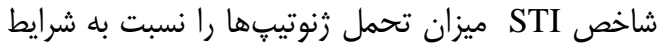

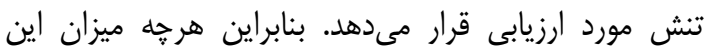

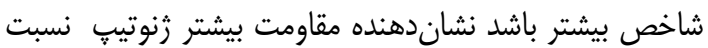

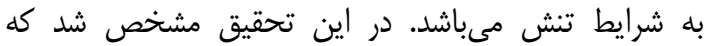
خنوتيبهاى C.H.T.209.12exF.K.40- C.H.209.12e

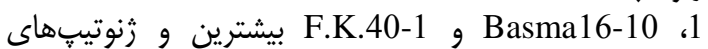
،SPT 433 ،SPT 432 sPT 412 ،SPT 408 ،Matianus Salouch و SPT 413 SPT 439

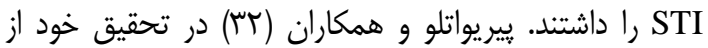

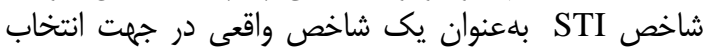

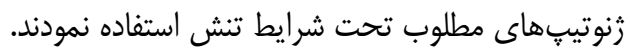
تجزيه كلاستر

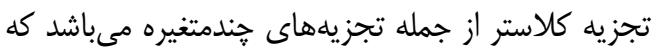

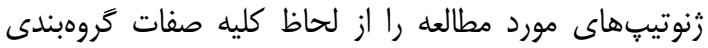

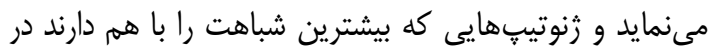

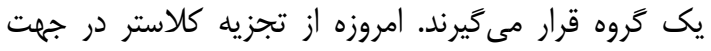

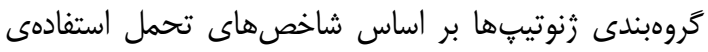

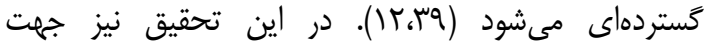

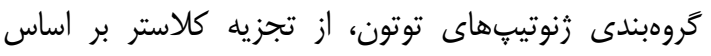

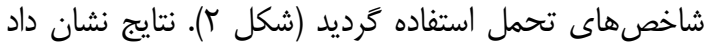

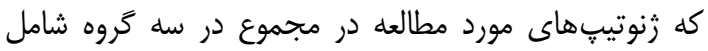

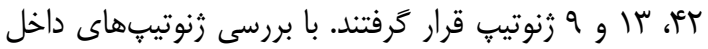

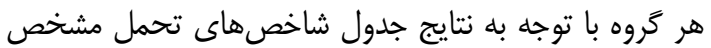

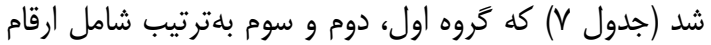

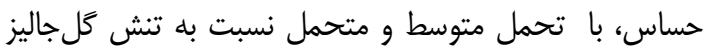

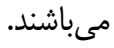
رنوتيبهايى كه ميزان SSI كمترى دارند به احتمال زياد

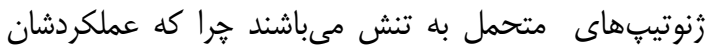

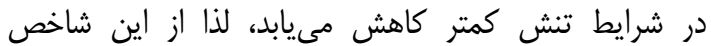

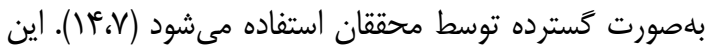

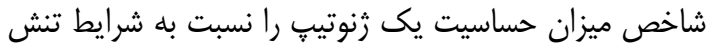

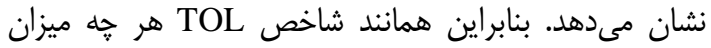

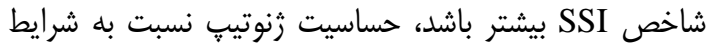

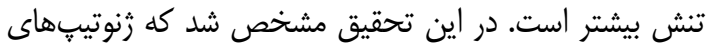

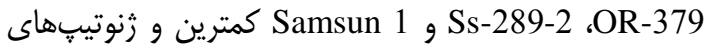

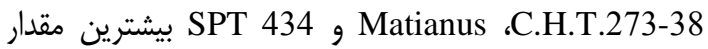
شاخص SSI را داشتند. كوتيرى و همكاران (19) از شاخص

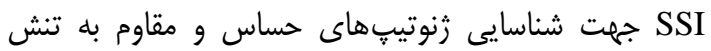

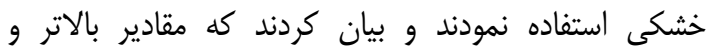

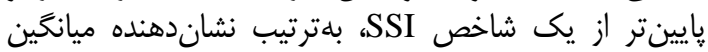

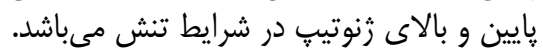

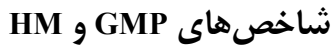

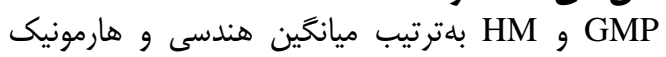

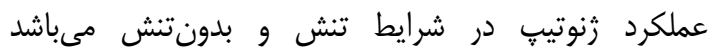

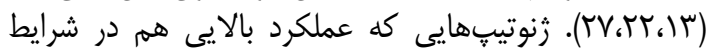

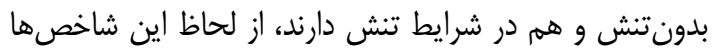

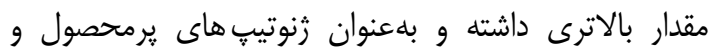

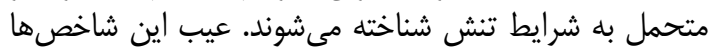

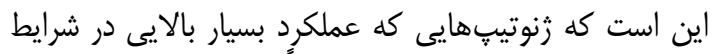

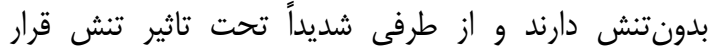

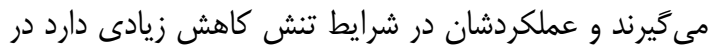

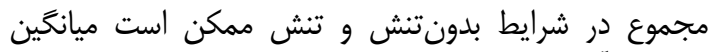

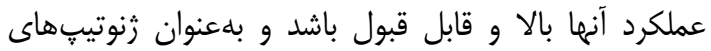

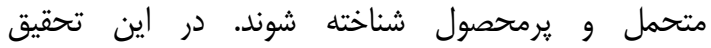
خنوتيبهاى C.H.T.209.12exF.K.40- C.209.12e

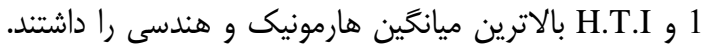

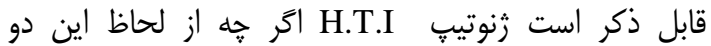

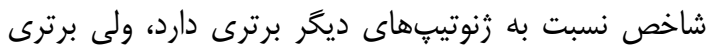

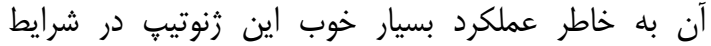

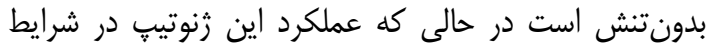
تنش به شدت كاهش يافته است. لذا اين رنوتيب رائ را فقط 
جدول V- ميانگين شاخصهاى تحمل به تنش و عملكرد تحت شرايط بدونتنش و تنش كل جاليز در زنوتيِهاى توتون Table 7. Mean of stress tolerance indices and yield under normal and orobanche stress conditions in tobacco

\begin{tabular}{|c|c|c|c|c|c|c|c|c|c|c|c|c|}
\hline خُ خُ جاليز & 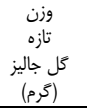 & جوانل جاليز & STI & HM & GMP & SSI & MP & TOL & 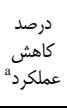 & $\begin{array}{l}\mathrm{Ys} \\
(\mathrm{g})\end{array}$ & $\begin{array}{l}\mathrm{Yp} \\
(\mathrm{g})\end{array}$ & رثنوتيب \\
\hline$r r / \Lambda \Lambda$ & $9 \pi / r \Lambda$ & $\Gamma / \Gamma$ & $\cdot / \mathrm{V}$ & $1 \% / r$ & $14 / 4$ & $1 / 8$ & $\Delta / \Delta$ & $11 / 1$ & $\Delta \Gamma / 91$ & $1.1 .^{\mathrm{n}-\mathrm{p}}$ & $r M / /^{b-j}$ & Kharmanli163 \\
\hline F/AT & $1 \% / 9 V$ & r r r & $\cdot / f$ & $N / 9$ & $9 / \Delta$ & $\cdot / V$ & $1 / r$ & $T / V$ & $T r / M$ & $q / 1^{1-p}$ & $11 / \mathrm{A}^{\mathrm{e}-\mathrm{J}}$ & Nevrokop \\
\hline$r / \Lambda$ & $19 / T \Delta$ & זr/r & $\cdot / f$ & 1.11 & $1 . / r$ & $\cdot / 1$ & $1 / 0$ & $r / 9$ & $r r / q V$ & $q / r^{1-p}$ & $|r /|^{e-j}$ & Trabozan \\
\hline.$/ r$ &.$/ 97$ &.$/ 0$ & $\mu / 1$ & $r / 1$ & $r V / \Lambda$ & $1 / 4$ & $V / \Delta$ & $10 / 1$ & $F \cdot / T V$ & $T r / \Psi^{a-1}$ & $r V / \Delta^{a-d}$ & Krumovgraid \\
\hline$s / r$ & ro/Aq & $r$ & $\cdot / 4$ & $q / 4$ & $1 . / 4$ & $. / 8-$ & $r / f$ & $f / \wedge$ & $r r / \Lambda$. & $q / f^{1-p}$ & $\mid f / r^{e-j}$ & Basma S. 31 \\
\hline או/או & $\Delta I / \Delta T$ & $r / \Delta$ &.$/ 9$ & $\mid \omega /$. & 181. &.$/ r$ & $\%$ & $N \cdot$ & $r V / r q$ & $\mid r / r^{T-p}$ & $r M / r^{D-J}$ & Triumph \\
\hline r & $r m / F$ & $r / \Delta$ &.$/ 1$ & $\Delta / \bar{q}$ & 91. & $1 / 1$ & $r / f$ & $F / q$ & $\Delta T / R Q$ & $k / r^{0-p}$ & $q / \Upsilon^{1-J}$ & Matianus \\
\hline$r / \Lambda r$ & $1 . / M$ & r & .18 & $1 r / 4$ & $I r / v$ &.$/ 9$ & $r / r$ & $r / s$ & $T N / F$. & $W / /^{t-p}$ & $\mid \varepsilon / \pi^{d-J}$ & Immni3000 \\
\hline S/Ir & 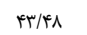 & r & $\cdot / r$ & $V / \Delta$ & $V / s$ & $\cdot / V$ &.$/ 9$ & $1 / 9$ & TI/AF & $\varepsilon / N^{\jmath-p}$ & $N / V^{t-j}$ & Tyk-Kula \\
\hline $1 Q / T Y$ & $\Delta F / q V$ & $r / 9 V$ & $\cdot / 1$ & $1 \% / 8$ & $10 / r$ & $\cdot / \Delta-$ & $\cdot / 1$ & $1 / 8$ & $9 / \Delta \Lambda$ & $\mid Q / /^{d-o}$ & $\mid \varepsilon / V^{c-J}$ & Ss-289-2 \\
\hline$s / v r$ & N &.$/ 0$ & $r / r$ & $\mathrm{rV} / \mathrm{q}$ & $r N / 1$ &.$/ \Delta$ & $1 / 9$ & $r / r$ & $1.19 \mathrm{~V}$ & $r \varepsilon / \Lambda^{a-c}$ & $r \cdot 10^{a-t}$ & Ohdaruma \\
\hline . & . & . & $1 / 1$ & $r / / r$ & $r r /$. &.$/ 8$ & $1 / v$ & $r / \Delta$ & $\mid f / \pi r$ & $r \cdot 1 \Delta^{\mathrm{b}-\mathrm{h}}$ & $r m / q^{b-j}$ & T.B.22 \\
\hline $1 / 4$ & $\Delta / / T$ & $1 / 9 \mathrm{~V}$ & .18 & $1 r / 9$ & $\pi / \mu$ &.$/ \pi$ & $\cdot / V$ & $1 / \Delta$ & $1 . / 45$ & $\mid r / q^{\mathrm{t}-\mathrm{p}}$ & $\mid F / e^{e-j}$ & Alborz 23 \\
\hline$s / \mathrm{VA}$ & $r \cdot / F$ & r & $\Gamma / \Lambda$ & гq/. & $r q / \Lambda$ & $1 / 1$ & $r / q$ & $\mathrm{~V} / \mathrm{V}$ & $r T / r r$ & $r \varepsilon / \Lambda^{a-c}$ & $r F / \Delta^{\mathrm{a}-\mathrm{e}}$ & F.K. $40-1$ \\
\hline s/Ir & TN/TV & . & $1 / 4$ & $M / V$ & $r \cdot / r$ & $1 / v$ & $\mathrm{~V} / \mathrm{\Lambda}$ & $10 / 9$ & $\Delta r / \Delta r$ & $|f /|^{\mathrm{e}-\mathrm{p}}$ & $r q / v^{a-g}$ & $\mathrm{Pl} 7$ \\
\hline $9 / 9$ & $r \Delta / \kappa r$ & 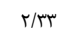 & $1 / 1$ & $18 / 9$ & $\mathrm{IV} / \Delta$ &.$/ 9$ & $r / V$ & $\Delta / f$ & $r \Delta / \Delta q$ & $1 \otimes / v^{d-1}$ & $r M / /^{b-j}$ & K.P.На \\
\hline // & $r / r$ & 1 &.$/ 9$ & $10 / \mathrm{V}$ & $\mid s / 1$ & $1 / 1$ & $r / r$ & $s / \Delta$ & $r r / 99$ & $\mid r / T^{t-p}$ & $19 / v^{c-1}$ & K.B. \\
\hline $.1 \cdot 0$ &.$/ \mathrm{VV}$ &.$/ 1 \mathrm{~V}$ & $r / r$ & $r / 1$ & $r m / 4$ &.$/ 4$ & $1 / v$ & $r / \Delta$ & $1 r / r q$ & $r \mathrm{r} / \mathrm{r}^{\mathrm{a}-\mathrm{g}}$ & $r \Delta / \kappa^{a-j}$ & G.D. 165 \\
\hline$r / \Delta T$ & 1.10 & $\cdot / 0$ & $r / 4$ & $r q / \Delta$ & rI/ & $1 / r$ & $9 / \Delta$ & $19 / 1$ & 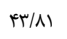 & $r F / a^{a-e}$ & $F \Psi / \gamma^{a-b}$ & H.T. I \\
\hline$\cdot$ & . & . & $\cdot / \Delta$ & $11 \%$ & $11 / v$ &.$/ 4$ & $1 / 9$ & $r / \wedge$ & $r \Delta / A V$ & $1 \cdot / 8^{\mathrm{h}-\mathrm{p}}$ & $|f /|^{e-j}$ & Kramograd N.H.H. 659 \\
\hline r & $9 / 1 \%$ & $r / \Delta$ &.$/ 8$ & $1 w / 1$ & $I r / v$ & $\cdot / \Lambda$ & $r / \Delta$ & $\Delta / 1$ & $r \cdot / \varphi q$ & $11 / \mathrm{r}^{\mathrm{t}-\mathrm{p}}$ & $\mid \varepsilon / \Lambda^{c-J}$ & T.K. 23 \\
\hline.$/ \Delta \Lambda$ & 1/9V &.$/ 9 V$ & $\cdot / \mu$ & $N / 9$ & $9 / \mathrm{V}$ & $\%$ & $1 / 9$ & $r / \wedge$ & $r \cdot / 4$. & $N v^{1-p}$ & $\mid r / \Delta^{e-j}$ & L16 \\
\hline$r / \Delta$ & $\mid r / v$ & 1 &.$/ 8$ & $11 / 9$ & $\mid r / \Lambda$ &.$/ r$ & $r / 9$ & $V / r$ & $4 . / 11$ & $1 \cdot / 8^{\mathrm{h}-\mathrm{p}}$ & $\mathrm{V} / \mathrm{v}^{\mathrm{c}-\mathrm{J}}$ & Izmir 7 \\
\hline $1 / 9 v$ & $F / T V$ &.$/ 9 V$ & $\cdot / \Delta$ & $1 . / 9$ & $11 / \Delta$ & $1 / r$ & $r / 9$ & $V / r$ & $F \Delta / \Delta V$ & $N / \xi^{1-p}$ & $10 / \Lambda^{a-j}$ & Pobeda 1 \\
\hline \multirow[t]{2}{*}{$r / 9$} & $\mid r / 4$ & $1 / \Delta$ & $\cdot / \mu$ & $N / \Delta$ & $N / 8$ & $\%$ & $1 /$ & $r / \cdot$ & r./gr & $\mathrm{V} / \mathrm{v}^{\mathrm{J}-\mathrm{p}}$ & $q / v^{t-j}$ & Rustica \\
\hline & & & $1 / 8$ & $19 / 1$ & $r \cdot / f$ & $\cdot / \Delta-$ & $1 / 9$ & $r / r$ & $1 \pi / N r$ & $19 / \Delta^{\mathrm{c}-1}$ & $r T / /^{\mathrm{b}-\mathrm{J}}$ & Samsun 1 \\
\hline \multirow[t]{2}{*}{$r / r \Delta$} & $N / 1 Q$ & 1 & $1 / r$ & $19 / 9$ & $\mathrm{IV} / \mathrm{\Lambda}$ & $1 / 1$ & $\Delta / r$ & $1 . / v$ & $\kappa r / Q 1$ & $\mid r / a^{t-p}$ & $r F / /^{\mathrm{D}-\mathrm{J}}$ & Samsun 959 \\
\hline & & & $1 / \Delta$ & $r \cdot / r$ & $r \cdot / q$ & $1 / r$ & $r / v$ & $9 / 4$ & rه/gr & $\mid \varepsilon / \Lambda^{c-k}$ & $r \varepsilon /\left.\right|^{a-j}$ & Samsun Katerini \\
\hline$I T / T r$ & is & ו/ג & $\cdot / 0$ & $\mid r / 1$ & $T / r$ &.$/ 9$ & $1 / 9$ & $r / 9$ & $r g / r q$ & $1 \cdot / 8^{\mathrm{h}-\mathrm{p}}$ & $\mid f / e^{e-j}$ & OR-205 \\
\hline$F / \Delta \Lambda$ & 19 & $r / I V$ &.$/ \mu$ & $N / \Lambda$ & $N / 9$ &.$/ 1$ &.$/ 4$ &.$/ 1$ & $V / \& \Delta$ & $N / v^{1-p}$ & $9 / 4^{1-J}$ & OR-345 \\
\hline$\cdot / 4$ & $1 / 90$ & r & $\cdot / r$ & $s / 4$ & s/8 & $1 / \%-$ &.$/ 1$ &.$/ r$ & $r / N G$ & $s / N^{J-P}$ & $\mathrm{~V} / \mathrm{g}_{\mathrm{g}-\mathrm{J}}$ & OR-379 \\
\hline 年 & $1 / 8$ &.$/ \Delta$ & $F / \Lambda$ & 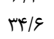 & r &.$/ 9$ & $v / v$ & $\mid Q / \Delta$ & 海 & $r r / *^{\mathrm{a}}$ & $\mathrm{F} / \mathrm{Q}^{\mathrm{a}}$ & C.H.T.209.12e \\
\hline$F / T$ & $F / T r$ & . & $f / f$ & $r / q$ & $r r / r$ &.$/ 9$ & $4 / 1$ & $N /$ & $r / \cdot r$ & $r \cdot / \Lambda^{a-b}$ & $\mathrm{rq} / \mathrm{s}^{\mathrm{a}-\mathrm{c}}$ & C.H.T.209. $12 \mathrm{e} \times$ F.K. $40-1$ \\
\hline$F / \Delta \Lambda$ & $18 / \wedge \Delta$ &.$/ \Delta$ & $\cdot / \mu$ & $q / r$ & $9 / r$ & $\cdot / \Delta$ & $\cdot / 4$ & $\cdot / 1$ & $\mathrm{~V} / \mathrm{If}$ & $q / 1^{1-p}$ & $9 / \Lambda^{t-j}$ & С.Н.Т. 266-6 \\
\hline $1 / v \wedge$ & $s / r \Delta$ & 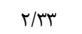 & $1 / 4$ & $\mathrm{IV} / \mathrm{V}$ & 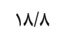 & $1 / 4$ & $r / \Lambda$ & $9 / v$ & rN/A. & $1 \Delta / /^{d-n}$ & $r \Delta / \mathrm{a}^{\mathrm{a}-\mathrm{J}}$ & С.Н.T. 283-8 \\
\hline $11 / \Delta \Delta$ & $\mu f / \cdot \Lambda$ & -/Ar & $1 / \Lambda$ & $19 / 9$ & $T r / \Delta$ & $r / \cdot$ & $\pi / 1$ & $T F / T$ & $9 \% / .4$ & $1 f / r^{e-p}$ & $r N / \psi^{a-a}$ & C.H.T.273-38 \\
\hline$r / 9 V$ & $\Delta / 9 \Delta$ & $1 / 9 V$ & $\mu / 1$ & $r q / 1$ & $r \varepsilon / V$ &.$/ 0$ & $r / r$ & $F / \Delta$ & $1 \omega / r$. & $r \Delta / 1^{a-a}$ & $r q / \xi^{a-n}$ & Basma 12-2 \\
\hline$T / 1 \Delta$ & $V / 10$ & 1 & $r / 9$ & $r \Delta / 8$ & re/. &.$/ 4$ & $\cdot / r$ & $\cdot / \Delta$ & $\mid / \wedge 1$ & $\mathrm{rV} / \mathrm{T}^{\mathrm{a}-\mathrm{c}}$ & $r V / \mathrm{N}^{\mathrm{a}-1}$ & Basma 16-10 \\
\hline F/T & $\mathrm{V} / \mathrm{\Lambda}$ & $1 / 1 V$ & $1 / 4$ & $N / r$ & $1 / \Lambda$ &.$/ 8$ & $r / r$ & $r / 8$ & $r \cdot / \mathrm{Ne}^{-}$ & $I V / T^{c-J}$ & $r M / v^{D-J}$ & Basma 104-1 \\
\hline $1 / N$ & $V / M$ & $1 / 9 V$ &.$/ \Delta$ & $11 / r$ & $11 / 9$ &.$/ 9$ & $1 / 1$ & $r / 8$ & $r \Delta / \mathrm{r}$ & $1 \cdot / \mathrm{f}^{\mathrm{h}-\mathrm{p}}$ & $\mid f / e^{e-j}$ & Basma 181-1 \\
\hline T/Tr & $r / \notin V$ & $1 / / V$ & $\cdot / 4$ & $9 / 1$ & $1 . / 4$ &.$/ 8$ & $r / f$ & $f / V$ & $r \Delta / \cdot v$ & $N / v^{1-p}$ & $\mid r / e^{e-j}$ & P.D. 325 \\
\hline.$/ r V$ & ./AV & r/או & $\cdot / r$ & $V / q$ & $\mathrm{~N} \cdot$ &.$/ \mu$ &.$/ 4$ &.$/ 9$ & $1 . / 4 V$ & $\mathrm{~V} / \mathrm{v}^{\mathrm{J}-\mathrm{p}}$ & $N / \mathcal{q}^{\mathrm{I}-\mathrm{J}}$ & P.D. 406 \\
\hline$F / \pi \Delta$ & $\mid F / \notin \Delta$ & $r / / r$ &.$/ 8$ & $1 \% /$. & $1 T / r$ & $\%$ &.$/ 9$ & $1 / r$ & $N / \Delta V$ & $I r / \Lambda^{t-p}$ & $\mid f / e^{e-j}$ & P.D. 328 \\
\hline$F / \Delta$ & $\mid g / 4 r$ & $1 / \Delta$ & $\cdot / 4$ & $1 . \%$ & $1 . / 4$ & $1 / r$ & $r / 9$ & $\Delta / \Gamma$ & $r q / \wedge \Delta$ & $N \cdot *^{\mathrm{J}-\mathrm{p}}$ & Ir/ J/R & P.D. 329 \\
\hline $1 / 91$ & $1 / 4 \wedge$ & r/r & $\cdot / \Delta$ & $I T / r$ & $I T / \Delta$ &.$/ 4$ & $1 / r$ & $r / \Delta$ & $\mid V / . r$ & $W / v^{t-p}$ & $|f /|^{e-j}$ & P.D. 336 \\
\hline.$/ E V$ & $1 . / r$ & $1 / M V$ & $\cdot / r$ & s/. & $s / \Delta$ & $1 / \cdot$ & $1 / 8$ & $r / r$ & $r V / r I$ & $\Delta / \digamma^{l-p}$ & $N / \xi^{t-j}$ & P.D. 345 \\
\hline $1 . / T V$ & $r r / 1$ & r & $\cdot / r$ & $\mathrm{~V} / \cdot$ & $\mathrm{V} / \mathrm{v}$ & $1 / \Delta$ & $r /$. & $8 / 1$ & $\Delta T / I F$ & $\Delta / \xi^{1-p}$ & $W / /^{t-j}$ & P.D. 371 \\
\hline$r / 9 v$ & س & r & $\cdot / 4$ & $11 / r$ & $11 / 4$ &.$/ r$ &.$/ r$ &.$/ V$ & $\Delta / M$ & $11 / r^{g-p}$ & $11 / a^{e-j}$ & P.D. 381 \\
\hline$F / T \Lambda$ & rI/ar & $1 / \Delta$ & $\cdot / r$ & $9 / 0$ & $s / \Delta$ &.$/ r$ & $\cdot / r$ &.$/ 8$ & $\mathrm{~N} / \mathrm{v}$. & $g / \mu^{k-p}$ & $g / 9^{\mathrm{h}-\mathrm{J}}$ & SPT 403 \\
\hline N/T & rז/אץ & r &.$/ 1$ & $\Delta / r$ & $\Delta / 8$ & $1 / 1$ & $1 / r$ & $r / 8$ & Tr/at & $F / \Delta^{\mathrm{n}-\mathrm{p}}$ & $\mathrm{V} / \mathrm{s}^{\mathrm{g}-\mathrm{J}}$ & SPT 408 \\
\hline$\Delta / T \Lambda$ & r./91 & I/ג & $\cdot / 1$ & $\varphi / \Delta$ & $F / \Delta$ &.$/ 8$ & $\cdot / 4$ &.$/ 9$ & $\mathrm{~W} / . \cdot$ & $F / /^{\mathrm{P}}$ & $\Delta / 0^{1-\mathrm{J}}$ & SPT 412 \\
\hline$v / N$ & rI/ar & $r / \Delta$ & $\cdot / r$ & $8 / 9$ & $V / r$ &.$/ 9$ & $1 / v$ & $r / 4$ & 的/QS & $\Delta / q^{1-p}$ & $q / \Psi^{t-j}$ & Isfehan5 \\
\hline$r / \Delta \Lambda$ & Ir/gr & 1 & $\cdot / 1$ & $\Delta / \Delta$ & $\Delta / q$ & $1 / 9$ & $r / r$ & $f / s$ & $\Delta T / A r$ & $F / l^{p}$ & $N / V^{t-j}$ & SPT 432 \\
\hline r/qr & $V / \Delta \Delta$ & I/ג & $\cdot / 1$ & $e / f$ & $f / f$ &.$/ 1$ &.$/ 1$ & $\cdot / \mu$ & S/Qr & $f / r^{0-p}$ & $F / \varepsilon$ & SPT 433 \\
\hline V/lr & $T V / q D$ & $r / 9 V$ &.$/ 0$ & $1 . / 9$ & $\mathrm{Ir} /$. & $1 / 1$ & $\Delta / 1$ & $1 . / 4$ & $\Delta \Delta / \cdot \Lambda$ & $N / e^{j-p}$ & $\mathrm{~W} / \mathrm{v}^{\mathrm{c}-\mathrm{J}}$ & SPT 434 \\
\hline$r / \Delta V$ & $r \Delta / q$ & r &.$/ 1$ & $\Delta / r$ & $\Delta / \Delta$ & $1 / \cdot$ & $1 /$ & $T / 1$ & $r / / r F$ & $f / s^{n-p}$ & $s / V^{1-3}$ & SPT 439 \\
\hline 海 & $8 / 9 \mathrm{~V}$ & 1 & $\cdot / r$ & $V / s$ & $N$ &.$/ 1$ & $1 / 1$ & $r / r$ & $r r / e r$ & $V / \omega^{j-p}$ & $9 / 1^{t-1}$ & SPT 441 \\
\hline$r / T V$ & $\mathrm{IV} / \mathrm{V}$ & $1 / 9 V$ &.$/ 1$ & $r / q$ & $f / F$ &.$/ 9$ & $1 / 9$ & $r / r$ & $\kappa q / \pi \Delta$ & $r / \digamma^{\mathrm{P}}$ & $s / v^{1-J}$ & SPT 413 \\
\hline.$/ 1 F$ & $1 / T V$ & r &.$/ 0$ & $11 / 1$ & $11 / 0$ & $1 /$ & $r / 1$ & $F / r$ & $r \cdot / v 1$ & $q / v^{\mathrm{h}-\mathrm{p}}$ & $\mid F / e^{e-j}$ & L17 \\
\hline r & . &.$/ 9 V$ &.$/ 1$ & $\Delta / 1$ & $\Delta / 1$ & $\cdot / \Delta$ & $\cdot / 4$ & $\cdot / 1$ & $|s| \cdot v$ & $\mathrm{f} / \mathrm{v}^{\mathrm{m}-\mathrm{p}}$ & $\Delta / \mathcal{A}^{-1-J}$ & Balouch \\
\hline$T / F V$ & $11 / \wedge \mu$ & r & $\cdot / r$ & $s / V$ & $\mathrm{~V} / \mathrm{l}$ & $1 / r$ & $1 / 9$ & $r / v$ & $\mathrm{rq} / \mathrm{v} \wedge$ & $\Delta / \mathcal{\zeta}^{1-p}$ & $9 / r^{t-j}$ & Saderati \\
\hline $1 / 1$ & s/Ar &.$/ 8 V$ & $\cdot / \mu$ & $9 / 1$ & $9 / \Delta$ & $1 / 4$ & $r / \Lambda$ & $\Delta / \Delta$ & Fr/TI & $v / r^{3-p}$ & $I T / v^{e-j}$ & Shahroudi \\
\hline s/1 & $r / \Delta$ & r & $\cdot / r$ & $9 / 9$ & $\mathrm{~V} / \mathrm{V}$ & $1 / r$ & $r / q$ & $\Delta / V$ & FN/VT & q/. k-p & $11 / v^{t-j}$ & T.K. L \\
\hline . & $1 / N r$ &.$/ 9 V$ & $1 /$ & $18 / \pi$ & $19 / 9$ &.$/ \Delta$ & $1 / r$ & $r / 9$ & $\mid F / A F$ & $1 \Delta / \omega^{\mathrm{d}-\mathrm{m}}$ & $W / T^{C-J}$ & C.Н.T. $269-12 \mathrm{e}$ \\
\hline
\end{tabular}




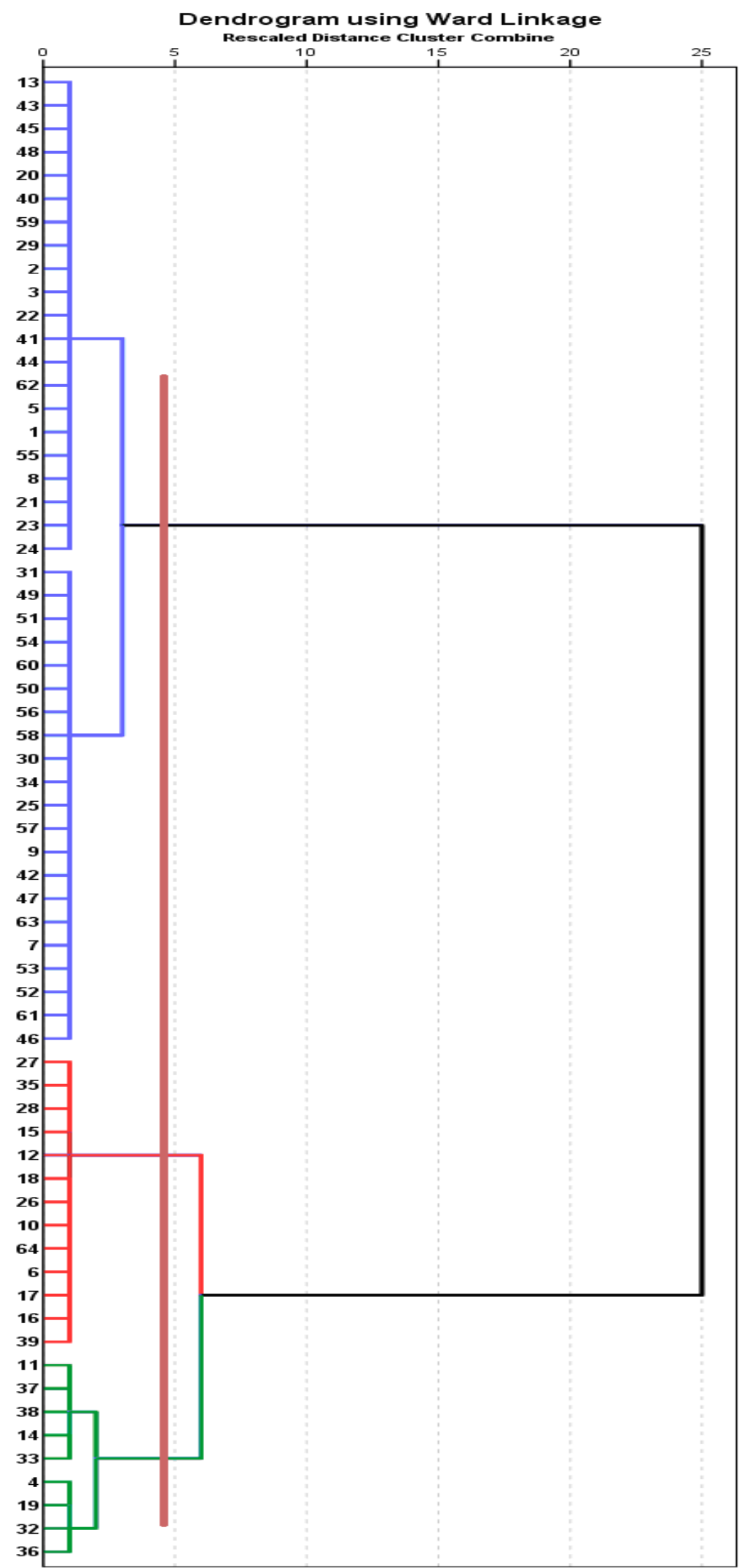

شكل r- تجزيه كلاستر بر اساس شاخصهاى تحمل به تنش و عملكرد زنوتيڤهاى توتون در شرايط بدونتنش و شرايط تنش كل جاليز.

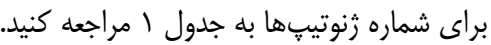

Figure 2. Cluster analysis based on stress tolerance indices and yield of tobacco genotypes under normal and orobanche stress conditions. For genotype code see Table 1. 


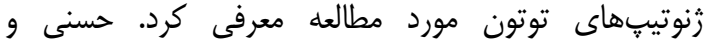

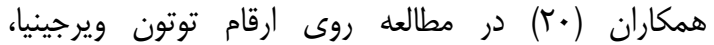

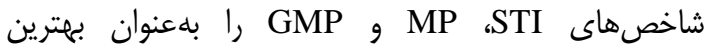

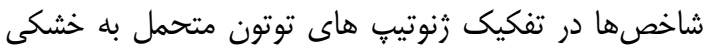

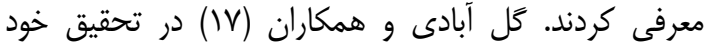

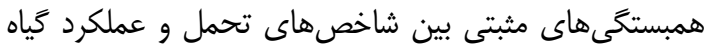

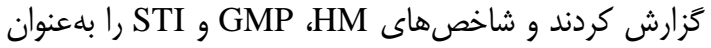

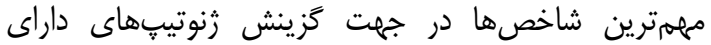

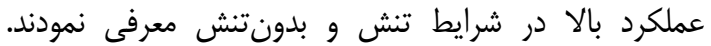

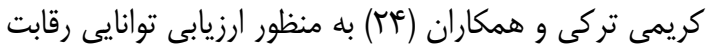

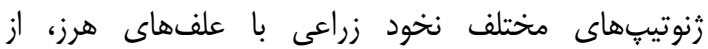
شاخصهاى تحمل تنش استفاده كردند. براساس نتايج تجزئ عزيه

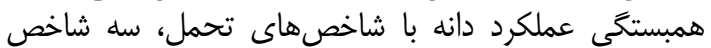

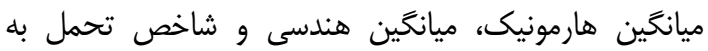

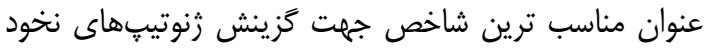
متحمل به تنش علف هرز معرفى شدند.
ضرايب همبستكى بين شاخص هاى تحمل

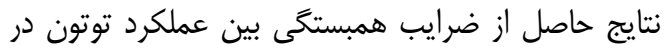

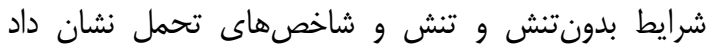

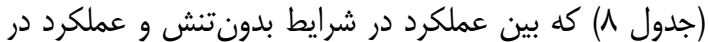

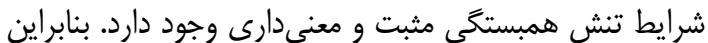

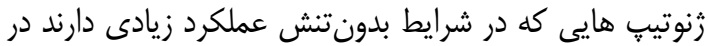

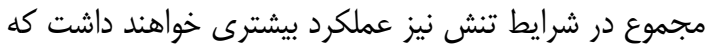

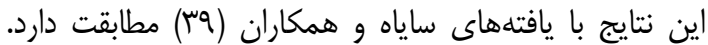

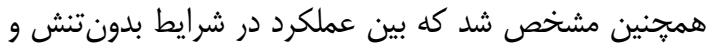

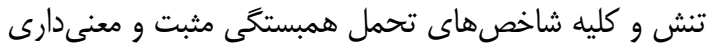

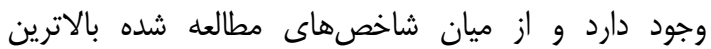

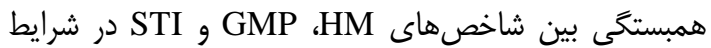

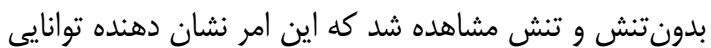

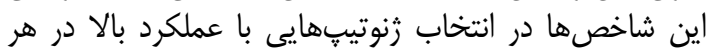

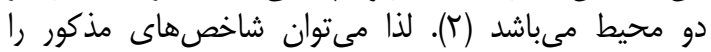
بهعنوان بهترين شاخصهاى ارزيابى تحمل به تنش مان در مران

جدول ^- ضر ايب همبستخى مابين شاخصهاى تحمل به تنش گل جاليز و عملكرد در شرايط بدونتنش و تنش در زنوتيِهاى توتون Table 8. Correlation coefficients among stress tolerance indices and yield under normal and orobanche stress conditions in tobacco genotypes

\begin{tabular}{|c|c|c|c|c|c|c|c|}
\hline & & & & & 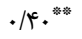 & $\cdot / V 9^{* * * 2}$ & TOL \\
\hline & & & $.19 . *$ & $\cdot 19 *^{*}$ & $-\cdot / \cdot r$ & $\cdot / T V^{*}$ & SSI \\
\hline & & זו/. & 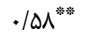 & 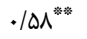 & 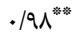 & $\cdot / 9 V^{* * * 3}$ & GMP \\
\hline & $\cdot / 98^{*}$ &.$/ 11$ & $\cdot / \uparrow \Lambda^{*{ }^{*}}$ & 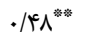 & 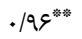 & 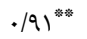 & STI \\
\hline
\end{tabular}

بيان نمود كه اين شاخصها مهمترين معيارها جهت انتخاب

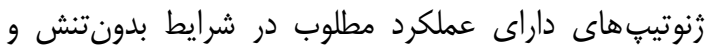

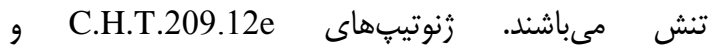
، C.H.T.209.12exF.K.40-1

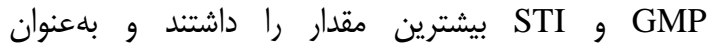

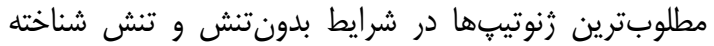

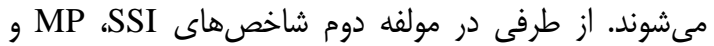

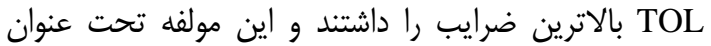
حساسيت نام گرفت و زنوتييهاى SPT 433

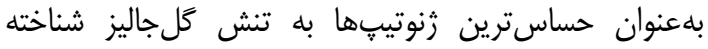
شدند.

\section{تجزيه به مولفههاى اصلى إنى}

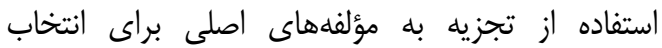

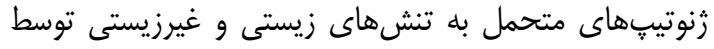

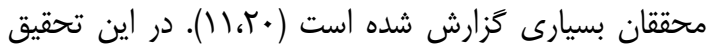

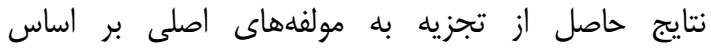

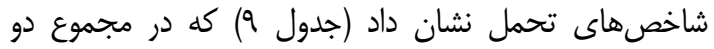

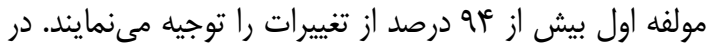

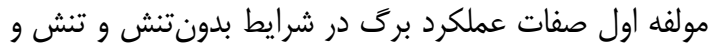

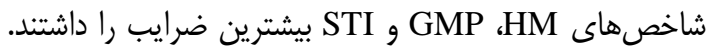
بنابراين اين مولفه تحت عنوان مولفه

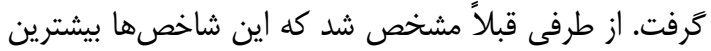

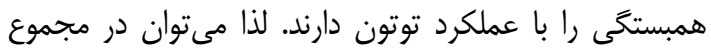

جدول q- تجزيه به مولفههاى اصلى براى عملكرد در شرايط بدونتنش و تنش گل جاليز و شاخصهاى تحمل به تنش Table 9. Principal component analysis for potential yield (YP), yields under Orobanche stress condition (YS) and

\begin{tabular}{|c|c|c|c|c|c|c|c|c|c|c|}
\hline مقدار ويزه & درصد تجمعى & HM & STI & GMP & SSI & MP & TOL & YS & YP & اجزاء \\
\hline$\Delta / V$ & $8 \mid /{ }_{1}$ &.$/ 91$ &.$/ 99$ & $\cdot / 9 \mathrm{~V}$ & -.01 .9 & . $/$ qq & $\cdot / \% q$ &.$/ 99$ &.$/ 199$ & 1 \\
\hline $1 / 1$ & $q F / \pi T$ & .119 &.$/ 19$ & $\cdot / F^{2}$ & $\cdot / \Lambda \mathcal{A}$ &.$/ 19$ & $\cdot / 199$ & $.1 \cdot r$ &.$/ 40$ & $r$ \\
\hline
\end{tabular}




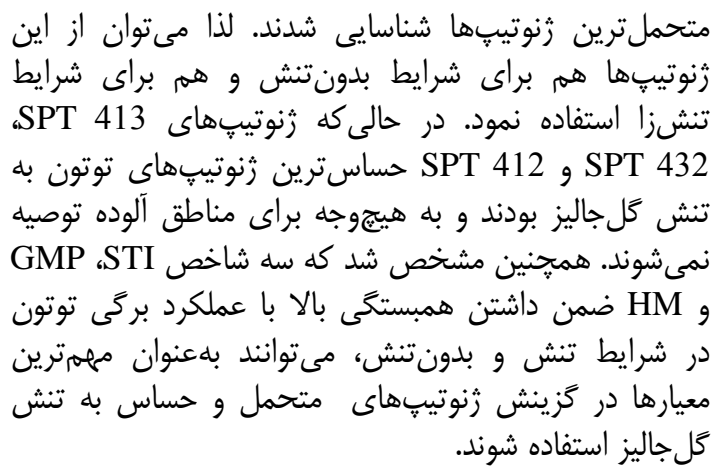

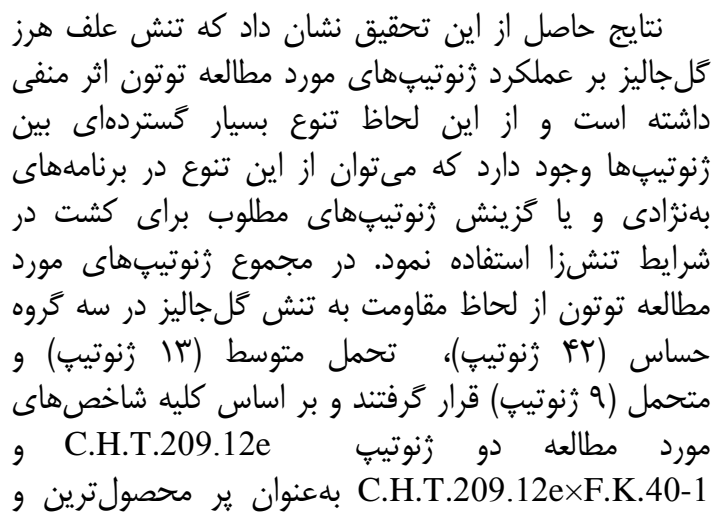

منابع

1. Abdollahi, A.S. and R. Noor Mohammad. 2007. Evaluation of bread wheat genotypes in terms of response to weed interference in dry conditions, Journal of Science and Technology of Agriculture and Natural Resources, 42(11): 93-102.

2. Blum, A. 1988. Plant breeding for stress environments. CRC press. Boca Raton, FL. 223 pp.

3. Bouslama, M. and W.T. Schapaugh. 1984. Stress tolerance in soybean. I. Evaluation of three screening techniques for heat and drought tolerance. Crop Science, 24: 933-937.

4. Bozhinova, R.P. 2006. Coefficients for determination of the leaf area in three burley Tobacco varieties Journal Central European Agriculture, 7: 7-12.

5. Brandle, J. and D. Bai. 1999. Biotechnology: uses and applications in tobacco improvement. In Tobacco: Production, Chemistry and Technology; Davis, N., Ed.; Wiley-Blackwell: Oxford, UK, pp: 49-65.

6. Bukhat, N.M. 2005. Studies in yield and yield associated traits of wheat (Triticum aestivum L.) genotypes under drought conditions. M.Sc. Thesis, Dissertation Submitted to Department of Agronomy, Sindh Agriculture University, Tandojam, Pakistan.

7. Clarke, J.M., R.M. De Pauwand and T.M. Townley-Smith. 1992. Evaluation of methods for quantification of drought tolerance in wheat. Crop Science, 32: 728-732.

8. Dalela, G.G. and R.L. Mathur. 1971. Resistance of varieties of eggplant, tomato and tobacco to broomrape (Orobanche cernua L.). Pest Articles and News Summaries, 17: 482-483.

9. Davalieva, K., I. Maleva, K. Filiposki, O. Spiroski and G.D. Efremov. 2010. Genetic Variability of Macedonian Tobacco Varieties Determined by Microsatellite Marker Analysis. Diversity, 2: 439-449

10. Dhanapal, G.N., P.C. Struik, M. Udayakumar and P. Timmermans 1996. Management of broomrape (Orobanche spp) - a review. Journal of Agronomy and Crop Science-Zeitschrift für Acker und Pflanzenbau, 176: 335-359.

11. Farshadfar, A., M. Zamani, M. Matlabi and A. Emamjome. 2001. Selection for drought resistance in chickpea. Iranian Journal of Agricultural Science, 32: 65-77.

12. Farshadfar, E. and J. Sutka. 2002. Multivariate analysis of drought tolerance in wheat substitution lines. Cereal Research Communications, 31: 33-39.

13. Fernandez, G.C.J. 1992. Effective selection criteria for assessing plant stress tolerance. In: Kuo, C.G. (Ed.), Adaptation of Food Crops to Temperature and water stress. pp: 257-269.

14. Fischer, R.A. and R. Maurer. 1978. Drought resistance in spring wheat cultivars. I. Grain yield responses. Australian Journal of Agricultural Research, 29: 897-912.

15. Forouzesh, S., M.A. Baghestani, H.M. Alizadeh, H. Rahimian Mashahadi and M. Minbashi Moini. 2008. Chemical control of orobanche (Orobanche aegyptiaca) in tomato. 2nd the Iranian Weed Science Congress, 29-30 January, Mashahad, 503-506 (In Persian).

16. Gavuzzi, P., F. Rizza, M. Palumbo, R.G. Campaline, G.L. Ricciardi and B. Borghi. 1997. Evaluation of field and laboratory predictors of drought and heat tolerance in winter and its components in wheat cultivars and landraces under near optimal and drought conditions. Euphytica, 113: 43-52.

17. Golabadi, M., A. Arzani and S.A.M. Mirmohamadi Maibody. 2006. Assessment of drought tolerance in segregating populations in durum wheat. African Journal of Agricultural Research, 1: 162-171.

18. Goldwasser, Y. and Y. Kleifeld. 2004. Recent approaches to Orobanche management: a review. In: Weed Biology and Management (ed. Inderjit), 439-466. Kluwer Academic Publishers, Dordrecht, Germany.

19. Guttieri, M.J., J.C. Stark, K.O. Brien and E. Souza. 2001. Relative sensitivity of spring wheat grain yield and quality parameters to moisture deficit. Crop Science, 41: 327-335. 
20. Hassani, S., H. Pirdashti, R. Mesbah and N. Babaeian Jelodar. 2008. Evaluation of drought tolerance indices in yield of six cultivars of Virginia tobacco (Nicotiana tabacum L.). Seed and Plant Production, 42: 129-144 (In Persian).

21. Darvishzadeh, R., M.J. Mousavi Andazghi and A. Fayyaz Moghaddam. 2017. Study on genetic of chlorine accumulation in leaves of oriental tobacco (Nicotiana tabacum L.). Journal of Crop Breeding, 9(22): 133-141 (In Persian).

22. Jafari, A., F. Paknejad and M. Jami Al-Ahmadi. 2009. Evaluation of selection indices for drought tolerance of corn (Zea mays L.) hybrids. International Journal of Plant Production, 3: 33-38.

23. Jebri, M., M. Ben Khalifa, H. Fakhfakh, B. Pérez-Vich and L. Velasco. 2017. Genetic diversity and race composition of sunflower broomrape populations from Tunisia. Phytopathologia Mediterranea, 56(3): 421-430.

24. Karimi Torki B., H. Hassanian Khoshro, M.R. Bihamta, P. Moradi and H. M. Alipour Yamchi. 2013. Evaluation of Tolerance of Chickpea Genotypes to Weed Competition. Seed and Plant Production Journal, 28(4): 471-487 (In Persian).

25. Karami-Nezhad, M. R., H. Rahimian Mashhadi, S. A. Siadat and M. Minbashi. 2005. Investigation on competition rye with wheat. First Iranian Congress of Weed Science, 24-25 Jan. 2005, Tehran, Iran. 276-279 (In Persian).

26. Khakwani, A.A., M.D. Dennett and M. Munir. 2011. Drought tolerance screening of wheat varieties by inducing water stress conditions. Songklanakarin Journal of Science and Technology, 33: 135-142.

27. Kristin, A.S., R.R. Serna, F.I. Perez, B.C. Enriquez, J.A.A. Gallegos, P.R. Vallejo, N. Wassimi and J. D. Kelley. 1997. Improving common bean performance under drought stress. Crop Science, 37: 43-50.

28. Mohsenzadeh Golfazani M., A. Aalami, H.A. Samizadeh, M. Shoaei Daylami and S. Talesh Sasani. 2012. Study of relationship between yield and yield components in tobacco genotype using path analysis method. Journal of Crop Breeding, 4(9): 26-40 (In Persian).

29. Munns, R. and R.A. James. 2003. Screening methods for salinity tolerance: A case study with tetraploid wheat. Plant and Soil, 253: 201-218.

30. Nazari, L. and H. Pakniyat, 2010. Assessment of drought tolerance in barley genotypes. Journal of Applied Sciences, 10: 151-156.

31. Oak, M., J. Basnayake, M. Tsubo, S. Fukai, K.S. Fischer, M. Cooper and H. Nesbittm. 2006. Use of drought response index for identification of drought tolerant genotypes in rainfed lowland rice. Field Crop Research, 99: 48-58.

32. Pireivatlou, A.S., B.D. Masjedlou and R.T. Aliyev. 2010. Evaluation of yield potential and stress adaptive trait in wheat genotypes under post anthesis drought stress conditions, African Journal of Agricultural Research, 5: 2829-2836.

33. Pujadas-Salva, A.J.O., J.A. Lopez Saez, P. Catalan and L.L. Saez. 2002. Plantas parásitas de la aleares ibérica e islas baleares. Mundi prensa, Madrid, pp: 345-451.

34. Ramirez-Vallejo, P. and J.D. Kelly. 1998. Traits related to drought resistance in common bean. Euphytica, 99: 127-136.

35. Roman, B., R. Hernandez, A.J. Pujadas-Salva, J.I. Cubero, D. Rubiales and Z. Satovic. 2007. Genetic diversity in two variants of Orobanche gracilis Sm. [var. gracilis and var. deludens (Beck) A. Pujadas] (Orobanchaceae) from different regions of Spain. Electronic Journal of Biotechnology, 10: 221-229.

36. Rosielle, A.A. and J. Hamblin, 1981. Theoretical aspects of selections for yield in stress and nonstress environments. Crop Science, 21: 943-946.

37. Rubiales, D. 2003. Parasitic plants, wild relatives and the nature of resistance. New Phytologist, 160: 459-461.

38. SAS Institute. 2002. SAS user's guide: Statistics version 9 for windows. SAS Institute, Carry, NC.

39. Sayyah, S.S., M. Ghobadi, S. Mansoorifar and A.R. Zebarjadi. 2011. Evaluation of Drought Tolerant in Some Wheat Genotypes to Post-anthesis Drought Stress. Journal of Agricultural Science, 4: 248256 (In Persian).

40. SPSS. SPSS software for windows version 21.0. Inc., Chicago, IL.

41. Valderrama, M.R., B. Román, Z. Satovic, D. Rubiales, J.I. Cubero and A.M. Torres. 2004. Locating quantitative trait loci associated with Orobanche crenata resistance in pea. Weed Research, 44: 323328 .

42. Vinogradov, V.A., E.K. Mironov, N.I. Strelyaeva and Y.F. Sarycher. 1981. Breeding tobacco for resistance to Orobanche. Tabak, 4: 55-57.

43. Zangi, M.R. 2005. Correlations between drought resistance indices and cotton yield in stress and nonstress condition. Asian Journal of Plant Science, 4: 106-108. 


\title{
Study of Tobacco (Nicotiana tabacum L.) Genotypes Reaction to Broomrape (Orobanche cernua L.) Weed Using Stress Tolerance Indices
}

\section{Reza Darvishzadeh $^{1}$ and Iraj Bernousi ${ }^{3}$}

1- Professor, Department of Plant Breeding and Biotechnology and Professor, Institute of Biotechnology, Urmia University, Urmia, Iran (Corresponding author: r.darvishzadeh@urmia.ac.ir)

3- Associate Professor, Department of Plant Breeding and Biotechnology, Urmia University, Urmia, Iran Received: July 24, 2018 Accepted: May 11, 2019

\begin{abstract}
In order to study the resistance of tobacco genotypes to Broomrape (Orobanche cernua L.), two experiments based on the randomized complete block design with three replications were conducted at non-stress and broomrape stress conditions at the tobacco research center of Urmia for two sucsessive years (2007-2009). The results showed that the broomrape infestation reduced the mean of all studied traits. The maximum reduction was recorded in aerial part dried weight $(31 \%)$ and root dried weight $(30 \%)$, respectively. So that, these traits were known as susceptible traits. The traits such as number of leaves, aerial part dried weight and root dried weight showed highly positive and significant correlation with tobacco yield at non-stress and stress conditions, respectively. So, one can use these traits for selecting tobacco genotypes with high yield performence. It was also found that there is a high correlation between the tobacco yield in non-stress and stress conditions with harmonic and geometric mean indices and stress tolerance index, so these indices can be used to assess tolerance to Broomrape stress in tobacco. Cluster analysis grouped the studied genotypes into three clusters containing susceptible genotypes (42 genotypes), moderately resistant (13 genotypes) and resistance (9 genotypes). In overall, the genotypes C.H.T.209.12e, C.H.T.209.12exF.K.40-1 and genotypes SPT413, SPT432 and SPT412 are introduced as the most resistance and the highest susceptible genotypes to Broomram, respectively.
\end{abstract}

Keywords: Cluster Analysis, Genetic Resistance, Oriental Tobacco, Parasitic Weed, Leaf Yield 